\title{
Efficient Global Aerodynamic Modeling from Flight Data
}

\author{
Eugene A. Morelli ${ }^{1}$ \\ NASA Langley Research Center, Hampton, Virginia, 23681
}

\begin{abstract}
A method for identifying global aerodynamic models from flight data in an efficient manner is explained and demonstrated. A novel experiment design technique was used to obtain dynamic flight data over a range of flight conditions with a single flight maneuver. Multivariate polynomials and polynomial splines were used with orthogonalization techniques and statistical modeling metrics to synthesize global nonlinear aerodynamic models directly and completely from flight data alone. Simulation data and flight data from a subscale twin-engine jet transport aircraft were used to demonstrate the techniques. Results showed that global multivariate nonlinear aerodynamic dependencies could be accurately identified using flight data from a single maneuver. Flight-derived global aerodynamic model structures, model parameter estimates, and associated uncertainties were provided for all six nondimensional force and moment coefficients for the test aircraft. These models were combined with a propulsion model identified from engine ground test data to produce a high-fidelity nonlinear flight simulation very efficiently. Prediction testing using a multi-axis maneuver showed that the identified global model accurately predicted aircraft responses.
\end{abstract}

\begin{tabular}{ll} 
& \multicolumn{1}{c}{ Nomenclature } \\
$a_{x}, a_{y}, a_{z}$ & $=$ body-axis translational accelerometer measurements, $\mathrm{g}$ \\
AirSTAR & $=$ Airborne Subscale Transport Aircraft Research \\
$b$ & $=$ wing span, $\mathrm{ft}$ \\
$\bar{c}$ & $=$ wing mean aerodynamic chord, $\mathrm{ft}$ \\
$C_{X}, C_{Y}, C_{Z}$ & $=$ body-axis nondimensional aerodynamic force coefficients \\
$C_{l}, C_{m}, C_{n}$ & $=$ body-axis nondimensional aerodynamic moment coefficients \\
$\mathrm{GPS}$ & $=$ global positioning system \\
$\mathrm{INS}$ & $=$ inertial navigation system \\
$I_{x}, I_{y}, I_{z}, I_{x z}$ & $=$ mass moments of inertia, slug- $\mathrm{ft}^{2}$ \\
$m$ & $=$ aircraft mass, slug \\
$M_{T}$ & $=$ body-axis pitching moment from engine thrust, $\mathrm{ft}-\mathrm{lbf}$ \\
$p, q, r$ & $=$ body-axis roll, pitch, and yaw rates, rad/s or deg/s \\
$\bar{q}$ & $=$ dynamic pressure, lbf/ $\mathrm{ft}^{2}$ \\
$S$ & $=$ wing reference area, $\mathrm{ft}^{2}$ \\
$\mathrm{SIDPAC}$ & $=$ System IDentification Programs for AirCraft \\
$T$ & $=$ maneuver length, sec \\
$T_{x}, T_{z}$ & $=$ body-axis engine thrust, lbf \\
$V$ & $=$ true airspeed, ft/s \\
$\alpha$ & $=$ angle of attack, rad or deg \\
$\beta$ & $=$ sideslip angle, rad or deg \\
$\delta_{e}, \delta_{a}, \delta_{r}$ & $=$ elevator, aileron, and rudder deflections, rad or deg \\
$\phi, \theta, \psi$ & $=$ Euler roll, pitch, and yaw angles, rad or deg \\
$\Sigma$ & $=$ covariance matrix \\
&
\end{tabular}

\footnotetext{
${ }^{1}$ Research Engineer, Dynamic Systems and Control Branch, MS 308, Associate Fellow
} 


$\begin{array}{lll}\frac{\text { superscripts }}{T} & \\ \cdot & =\text { transpose } \\ \cdot & =\text { estimate } \\ -1 & =\text { matrix inverse } \\ \text { subscripts } & \\ c g & =\text { center of gravity } \\ o & =\text { reference value or base term }\end{array}$

\title{
I. Introduction
}

\begin{abstract}
$\mathrm{A}$ CCURATE flight simulation has many important applications in aircraft dynamics and control, such as pilot training, mission rehearsal, dynamic analysis, and control system design ${ }^{1-3}$. Typically, flight simulations are based on wind tunnel data and/or results from computational methods ${ }^{2-8}$. Flight data are often used subsequently to modify or augment simulations for improved fidelity to the real flight vehicle ${ }^{9-12}$. Furthermore, the Federal Aviation Administration (FAA) requires flight data matching criteria to be satisfied as part of the certification process for commercial flight training simulators.
\end{abstract}

Resources are always limited, and wind tunnel testing and aerodynamic calculations require time and money. Building an aircraft simulation based only on flight data, without wind tunnel testing or aerodynamic calculations, could be effective for certain types of rapid, relatively low-budget research and development projects, such as subscale flying models and unmanned aerial vehicles (UAV). In such cases, there is no human life risked aboard the aircraft, and typically the aircraft are known to be flyable. The capability to build a high-fidelity nonlinear simulation based on a few test flights would be very beneficial in terms of project cost and schedule.

Using flight data to update a simulation database generated from wind tunnel data or aerodynamic calculations is often a time-consuming and iterative task done in an ad hoc way, although recent work has focused on automated simulation updating methods ${ }^{11,12}$. If an accurate global aerodynamic model could be generated from flight data alone, the problem of flight-updating a simulation database based on wind tunnel data or aerodynamic calculations could be avoided altogether. Even in cases where a simulation has been generated based on wind tunnel data and aerodynamic calculations, a flight-determined global aerodynamic model could be used for simulation validation, updating based on flight data, and quantifying the effects of Reynolds number and geometry differences between the full-scale flight vehicle and the wind tunnel test article.

In this work, a method for identifying a global aerodynamic model efficiently from flight data alone is examined. The method includes a novel approach to designing the maneuvers used to collect flight data for modeling purposes, and a novel approach to global aerodynamic modeling based on the flight data. The next section describes the maneuver design, which leverages past work on input design for real-time dynamic modeling ${ }^{13-17}$. Following this, the global aerodynamic modeling approach is described. The approach combines multivariate polynomials, polynomial splines, orthogonal function modeling theory, and statistical modeling metrics to identify global aerodynamic models for nondimensional force and moment coefficients. The novel flight test maneuver design and global aerodynamic modeling method are then demonstrated using simulated data and flight data from a subscale twin-engine jet transport aircraft. A flight simulation was created by combining previously developed simulation software $^{6,14}$ written in MATLAB ${ }^{\circledR}$ with the global aerodynamic model identified from flight data and a propulsion model identified from ground test data. A multi-axis doublet sequence maneuver executed at a single flight condition (nominal angle of attack) was used for prediction testing to evaluate the fidelity of the global aerodynamic models identified from flight data. Details of the flight test and prediction results are presented in Section VI.

The T-2 subscale jet transport aircraft used for this study, described in detail in Section IV, was also tested extensively in the wind tunnel ${ }^{5,8}$, although the wind tunnel test article differed in some geometric details from the aircraft used for the flight tests. The wind tunnel data provided an aerodynamic database for the nonlinear simulation, which was used to validate the effectiveness of the global aerodynamic modeling procedure using flight data. Comparisons using global aerodynamic models identified from flight data and the wind tunnel aerodynamic database are shown in Sections V and VI.

All of the experiment design, data analysis, and modeling tasks included in this work were done using system identification software written in MATLAB ${ }^{\circledR}$, called System IDentification Programs for AirCraft, or SIDPAC ${ }^{14}$. SIDPAC is bundled with Ref. [14], and is therefore publicly available. The SIDPAC software toolbox was 
developed at NASA Langley, and is continually expanded and improved. SIDPAC has been applied successfully to a wide variety of flight and wind tunnel experiments at NASA Langley ${ }^{14}$ and elsewhere, and is used at more than 80 institutions worldwide ${ }^{18}$.

\section{Maneuver Design}

\section{A. Orthogonal Optimized Multi-Sine Input Design}

This section describes how orthogonal optimized multi-sine inputs are designed and why this particular input form is efficient for identifying dynamic models from flight data. More details on this input design technique and flight applications can be found in Refs. [13]-[17].

The general idea is to excite the aircraft using perturbation inputs with wideband frequency content over a range of frequencies that encompasses the expected modal frequencies for the aircraft dynamic response. The excitations are implemented as perturbations to the control surface deflections by summing designed perturbation inputs with the actuator commands from the pilot and feedback control system, just before the actuator limiting on command rate and position.

Each designed perturbation input is a sum of sinusoids with unique frequencies, optimized phase shifts, and specified power distribution. Component frequencies are selected to cover a frequency band of interest, similar to frequency sweeps. The wide-band frequency content of the inputs is important because there is naturally some uncertainty as to what the modal frequencies are for the aircraft in flight. Wide-band inputs provide robustness to this uncertainty. Phase shifts for the sinusoidal components of each input are optimized to achieve low peak-to-peak amplitude and high input energy content for the sum of sinusoids. Amplitudes of the individual sinusoidal components can be chosen to achieve a specific power distribution.

Multiple inputs are designed to be mutually orthogonal in both the time domain and the frequency domain, and are optimized for maximum data information content in multiple axes over a short time period, while minimizing excursions from the nominal flight condition. The mutual orthogonality of the inputs allows simultaneous application of multiple inputs, which helps to minimize excitation time, but more importantly for this work, provides continuous multi-axis excitation as the aircraft flies through time-varying or precarious flight conditions.

Each perturbation input $\boldsymbol{u}_{j}$, which is to be applied to the $j^{\text {th }}$ individual control surface, is comprised of a set of summed harmonic sinusoids with individual phase shifts $\phi_{k}$,

$$
\boldsymbol{u}_{j}=\sum_{k \in\{1,2, \ldots, M\}} A_{k} \sin \left(\frac{2 \pi k \boldsymbol{t}}{T}+\phi_{k}\right) \quad j=1,2, \ldots, m
$$

where $M$ is the total number of available harmonically-related frequencies, $T$ is the time length of the excitation, and $A_{k}$ is the amplitude for the $k^{\text {th }}$ sinusoidal component. The variable $t$ represents a vector of $N$ discrete time points, $\boldsymbol{t}=\left[\begin{array}{llll}t(0) & t(1) \ldots t(N-1)\end{array}\right]^{T}$, and $\boldsymbol{u}_{j}$ represents the vector of corresponding amplitudes for the $j^{\text {th }}$ input, $\boldsymbol{u}_{j}=\left[\begin{array}{llll}u_{j}(0) & u_{j}(1) & \ldots & u_{j}(N-1)\end{array}\right]^{T}$. Each of the $m$ inputs is comprised of selected components from the pool of $M$ harmonic sinusoids with frequencies $\omega_{k}=2 \pi k / T, k=1,2, \ldots, M$, where $\omega_{M}=2 \pi M / T$ represents the upper limit of the frequency band for the excitation. The interval $\left[\omega_{1}, \omega_{M}\right] \mathrm{rad} / \mathrm{s}$ specifies the range of frequencies where the aircraft dynamics are expected to lie.

If the phase angles $\phi_{k}$ in Eq. (1) were chosen at random on the interval $(-\pi, \pi] \mathrm{rad}$, then in general, the various harmonic components would add together at some points to produce an input $\boldsymbol{u}_{j}$ with relatively large amplitude excursions. This is undesirable, because it can result in the dynamic system being moved too far from the reference condition selected for the experiment. To prevent this, the phase angles $\phi_{k}$ for the selected harmonic components are chosen to minimize relative peak factor $R P F^{14}$, defined by 


$$
R P F\left(\boldsymbol{u}_{j}\right)=\frac{\left[\max \left(\boldsymbol{u}_{j}\right)-\min \left(\boldsymbol{u}_{j}\right)\right] / 2}{\sqrt{2\left(\boldsymbol{u}_{j}^{T} \boldsymbol{u}_{j}\right) / N}}=\frac{\left[\max \left(\boldsymbol{u}_{j}\right)-\min \left(\boldsymbol{u}_{j}\right)\right]}{2 \sqrt{2} \operatorname{rms}\left(\boldsymbol{u}_{j}\right)}
$$

Relative peak factor is a measure of the efficiency of an input for dynamic modeling purposes, in terms of the amplitude range of the input divided by a measure of the input energy. The relative peak factor is scaled so that any individual sinusoidal component (such as any one of the summands in Eq. (1)) has $R P F=1$. Low relative peak factors are desirable and efficient for estimating dynamic model parameters, because the objective is to excite the dynamic system with good input energy over a variety of frequencies while minimizing the input amplitudes in the time domain, to avoid driving the dynamic system too far away from the reference condition.

For a composite signal with more than one sinusoidal component, as in Eq. (1), the goal of designing an input with minimum $R P F$ is achieved by adjusting the phase parameters $\phi_{k}$ for the sinusoidal components of the input. The resulting optimization problem is non-convex; however, a simplex algorithm ${ }^{14,19}$ can be applied to find a solution.

The integers $k$ specifying the frequencies for the $j^{\text {th }}$ input $\boldsymbol{u}_{j}$ are selected to be unique to that input, but are not necessarily consecutive. A good approach for multiple inputs is to assign integers $k$ to each input alternately. This is illustrated in Figure 1 for a flight test maneuver design on the T-2 subscale jet transport aircraft described in Section IV. In that case, there were 3 inputs: elevator, rudder, and aileron, and a total of 30 frequencies $(M=30)$. The frequencies were interleaved among the three inputs to achieve wide-band frequency content for each input. This provided robustness to uncertainty in how each control excites the dynamic modes of the aircraft. Because each input has wide-band frequency content, the same input design can be applied at various flight conditions, which simplifies the flight test and reduces flight computer memory requirements. It is even possible to use the same input design for different aircraft, because of the wide-band frequency content of the excitation inputs.

To achieve a uniform power distribution, the $A_{k}$ are selected as

$$
A_{k}=\frac{A}{\sqrt{n}} \quad \forall k
$$

where $n$ is the number of sinusoidal components included in the summation of Eq. (1) for $\boldsymbol{u}_{j}$, and $A$ is the amplitude of the composite input $\boldsymbol{u}_{j}$. Therefore, with uniform power distribution, selection of the $A_{k}$ reduces to selecting a single value for the input amplitude $A$. Each input $\boldsymbol{u}_{j}$ can of course have arbitrary amplitude $A$, subject to practical flight testing and modeling constraints.

It is also possible to modify the power at individual frequencies for each input, to focus the excitation on frequencies near where the natural frequencies of the dynamic modes are believed to be, or to avoid exciting structural responses, for example. For each input, the power spectrum can be tailored by selecting the $A_{k}$ in Eq. (1) to distribute power over the spectral components. The power spectra shown in Figure 1 are normalized, so the effects of individual control surface amplitudes are excluded. This means that for each input, the sum of all the spectral line ordinates (sum of the heights of the bars for each input) is 1 .

When the frequency indices $k$ selected for each

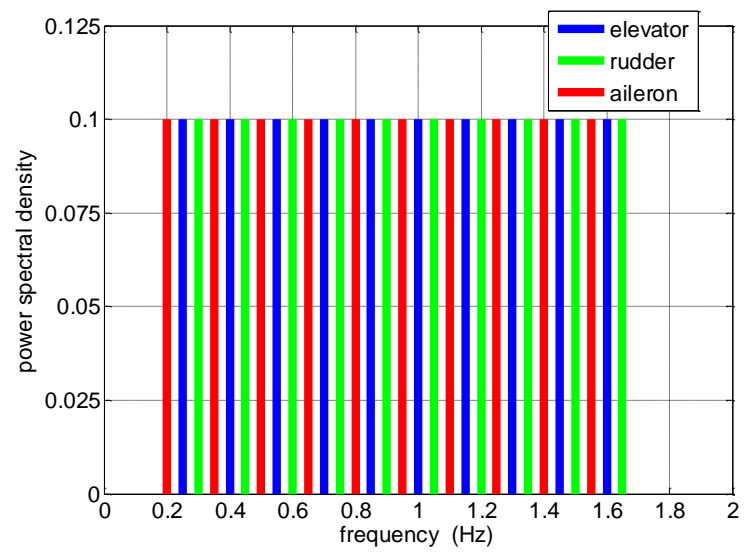

Figure 1. Multiple orthogonal phase-optimized multi-sine input spectra input $\boldsymbol{u}_{j}$ in Eq. (1) are distinct from those chosen for the other inputs, then the frequency content of each $\boldsymbol{u}_{j}$ consists of distinct spectral lines in the frequency domain, as can be seen in Figure 1. Therefore, the vectors of Fourier transforms for the inputs as a function of frequency have inner 
products equal to zero. In this sense, the inputs are mutually orthogonal in the frequency domain, because each input contains frequencies that are not present in the other inputs.

In the time domain, a sum of harmonic sinusoids is orthogonal to any other sum of sinusoids with harmonicallyrelated frequencies, regardless of the constant phase shift of each sinusoidal component ${ }^{14,15}$. Consequently, the inputs are also mutually orthogonal in the time domain.

An objective for the experiment design is to excite the aircraft dynamics in all axes over a short time period by moving multiple control surfaces simultaneously. This is particularly important in situations where the reference flight condition is time-varying, or cannot be maintained for very long.

Since more than one surface is being moved, it is advantageous for modeling purposes if the $\boldsymbol{u}_{j}$ applied to the control surfaces are mutually orthogonal. This helps the dynamic modeling by completely de-correlating the inputs,
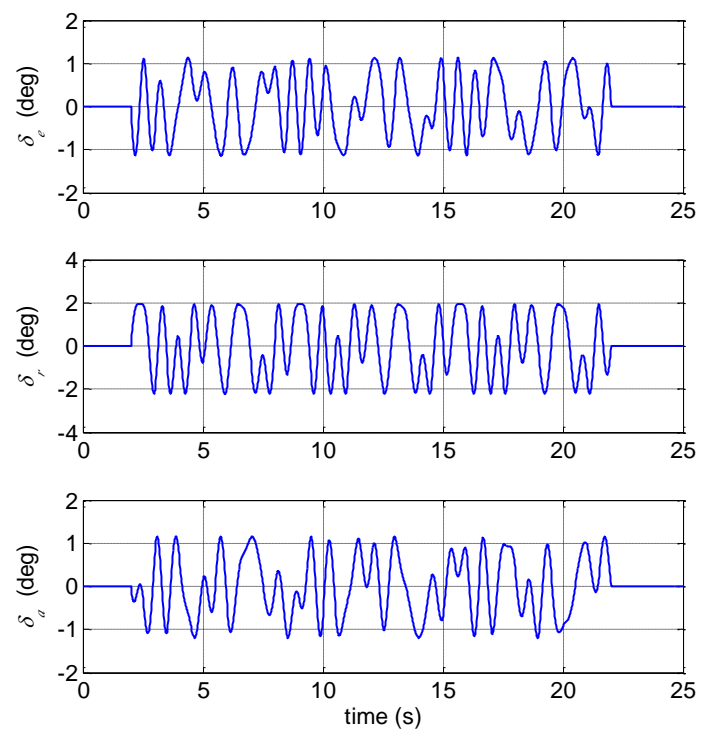

Figure 2. Multiple orthogonal phase-optimized multi-sine excitation inputs which improves the accuracy of control effectiveness estimates. Using the input design method described here, it is possible to make all of the $\boldsymbol{u}_{j}$ mutually orthogonal in both the time and frequency domains, while also minimizing relative peak factor for each $\boldsymbol{u}_{j}$, which keeps the aircraft from departing significantly from the reference flight condition. This gives the analyst the flexibility to use either time-domain or frequency-domain parameter estimation methods, while retaining the desirable feature of mutually orthogonal inputs.

Figure 2 shows the perturbation input time series for a maneuver design flown on the T-2 subscale jet transport aircraft. These inputs are mutually orthogonal in both the time and frequency domains. The inputs were computed from Eq. (1) and the information in Table 1, where the phase angles $\phi_{k}$ were optimized for minimum relative peak factor. Because of the various frequencies and phase angles, and the small amplitudes of the perturbation inputs, applying these inputs simultaneously to the aircraft produces a dynamic response similar to what might be seen in flight through light to moderate turbulence. The aircraft stays near the reference condition, but responds dynamically about that condition. In practice, pilot inputs and feedback control can act to ruin the input orthogonality; however, good modeling results require only low correlations, not zero correlations, so that slightly imperfect inputs still work quite well.

\section{B. Maneuver Design for Efficient Global Aerodynamic Modeling}

In past work ${ }^{13-17}$, inputs such as those shown in Figure 2 were applied at a selected flight condition. The pilot flew the aircraft to the reference flight condition, then activated an automated excitation system that added inputs like those shown in Figure 2 to the trim control deflections coming from the pilot and feedback control system. This produced excellent data for aerodynamic parameter estimation at the selected flight condition. This approach could be called local aerodynamic modeling.

For global aerodynamic modeling, one conventional approach is to combine local aerodynamic modeling results obtained from local perturbation maneuvers. This requires numerous and accurate acquisitions of particular flight conditions, followed by the application of perturbation excitations at each condition, and combining the local results to produce a global model.

More efficient global aerodynamic modeling can be achieved by continuously applying multi-axis perturbation inputs while the aircraft flight condition is varied slowly. This approach is practical because the orthogonal optimized multi-axis perturbations excite the aircraft dynamics in a very time-efficient manner with high data information content, so that the aircraft dynamics can be sufficiently excited even when the flight condition is changing.

To implement an efficient global aerodynamic modeling maneuver, the pilot began with a steady wings-level trim condition at low angle of attack, then initiated automated multi-axis excitation inputs like those shown in 
Figure 2. While the excitation inputs were being applied, the pilot pulled back slowly on the stick to induce a slow increase in the nominal angle of attack. The multi-axis excitation inputs were continuously applied additively to the control surface deflections commanded by the pilot and control system. Pilot inputs on lateral stick and rudder pedal were essentially zero, but the elevator deflection commanded by the pilot changed slowly to implement the slow increase in nominal angle of attack. An example of the resulting flight data is shown in Figure 3. This maneuver produces very informative data over a wide range of nominal angle of attack. Only subsonic aerodynamics at relatively low altitude were being studied, so the effects of changing airspeed were adequately modeled by
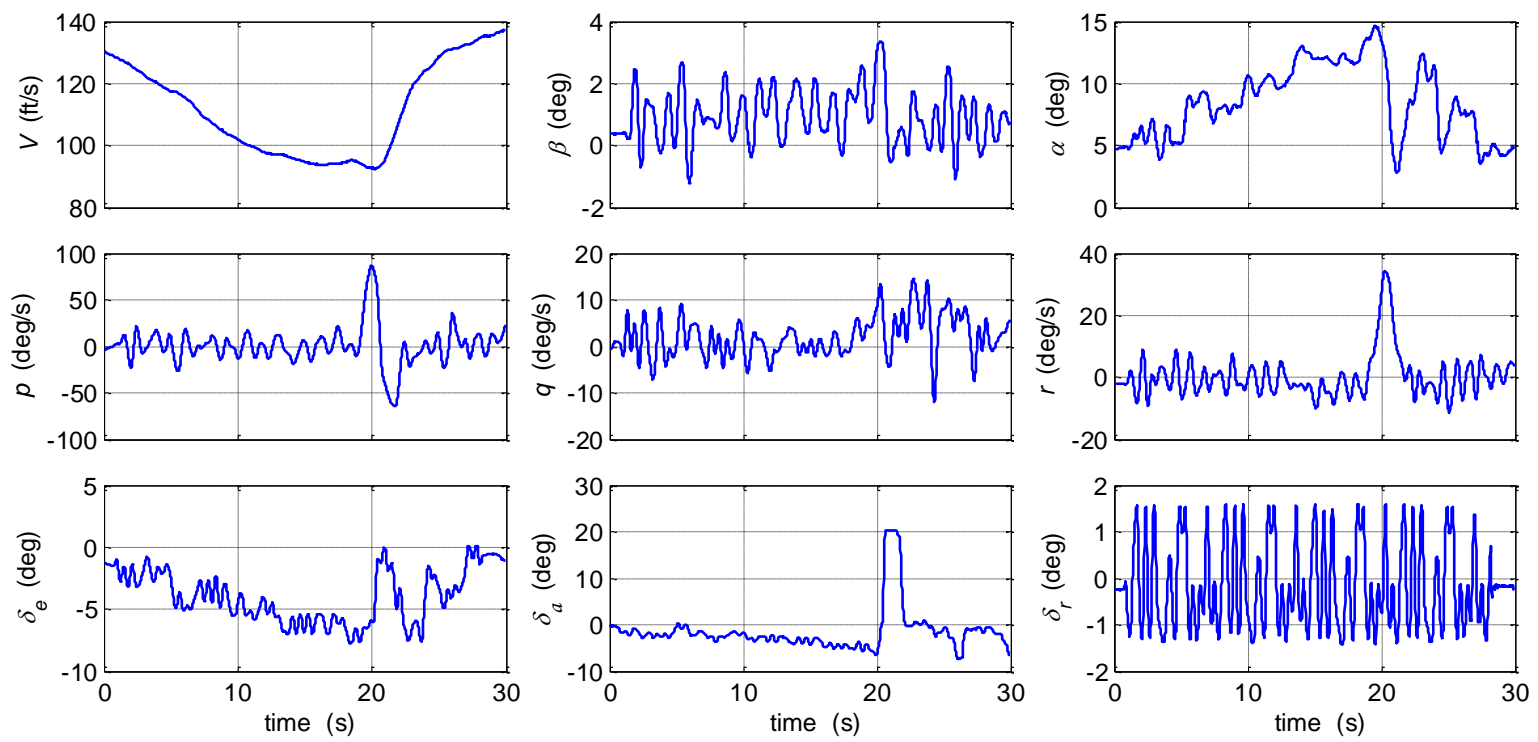

Figure 3. T-2 flight data for a global aerodynamic modeling maneuver

conventional nondimensionalization using dynamic pressure. Since the nominal angle of attack changed slowly, this maneuver can be considered a combination of informative multi-axis excitation data for many different nominal angles of attack, executed in a single, efficient, combined maneuver.

Figure 4 shows cross-plots of aircraft states and controls using data from the maneuver shown in Figure 3. These plots demonstrate that a wide range of the explanatory variables generally used for aerodynamic modeling was swept through during this single maneuver. Note also that the cross-plots generally do not show diagonal lines or ellipses, which means that the explanatory variable data from this maneuver had very low pair-wise correlations. Low pair-wise correlations mean that the aerodynamic dependencies on the explanatory variables can be identified accurately and without ambiguity. Cross-plots for other aircraft states and controls were similar in that the plots indicated low pair-wise correlations for the explanatory variables.
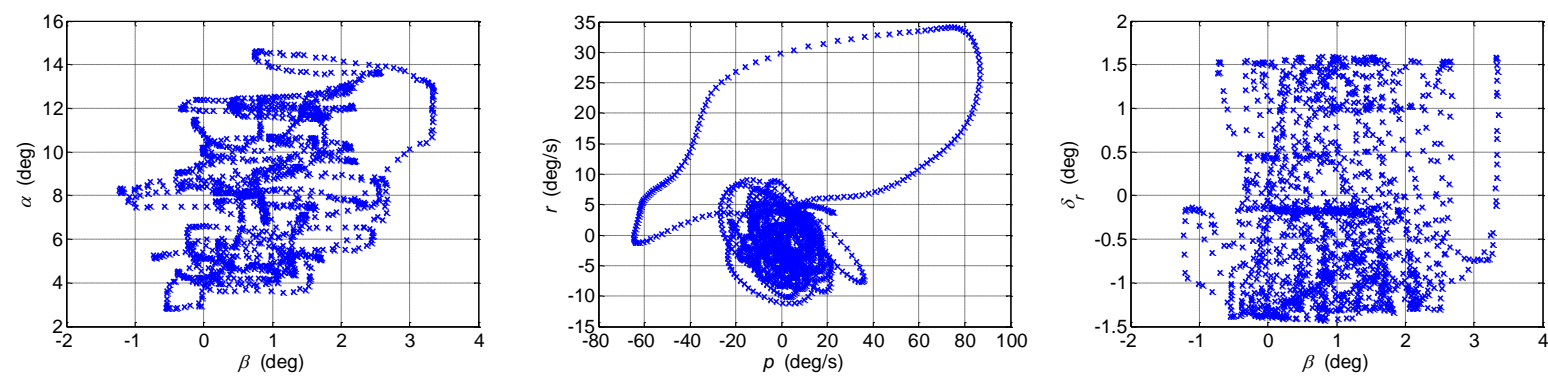

Figure 4. T-2 flight data cross plots for a global aerodynamic modeling maneuver

This novel maneuver exhibits a combination of low pair-wise correlations, multi-axis excitation, and slowlyvarying flight conditions that cover a large portion of the explanatory variable subspace for aerodynamic modeling. These characteristics make the maneuver very effective and efficient for global aerodynamic modeling. 
A series of these maneuvers were flown on the T-2 subscale jet transport aircraft. Each maneuver had a constant power setting and aircraft configuration, and all used multi-axis perturbation time series, similar to what is shown in Figure 2. The wide-band frequency content of the excitation inputs made them effective for a wide variety of flight conditions, power settings, and aircraft configurations. Over several flight test deployments, some variations in the frequency content, time length, and amplitudes of the excitation inputs were implemented, in order to study the effects of these variations from an experiment design standpoint. Table 2 lists the power setting and aircraft configuration for each maneuver in the series flown on the T-2 aircraft.

\section{Global Aerodynamic Modeling}

The objective of global aerodynamic modeling is to identify a model for each nondimensional aerodynamic force and moment coefficient as a function of explanatory variables that can be measured, such as angle of attack, pitch rate, and control surface deflections, over a large range of the explanatory variables. There are two main difficulties in doing this: 1) experiment design to collect dynamic data over a large range of the explanatory variables, which was addressed in Section II, and 2) identifying an accurate global model, which is the subject of this section.

In the typical approach to global aerodynamic modeling, a series of linear or simplified local models, which are valid for relatively small ranges of the explanatory variables, are joined together in some way to implement a global aerodynamic model. In another approach, a multivariate orthogonal function modeling technique was developed to identify global models, which were generally nonlinear and valid over relatively large ranges of the explanatory variables $^{20}$. However, for very large ranges of the explanatory variables, or severe local nonlinearities, this approach in some cases compromises local model fit in order to achieve a better global model fit. This is the result of using global orthogonal polynomial functions for the modeling, which do not have the ability to change locally without modifying the entire model. Other approaches have used data partitioning with simplified local model structures ${ }^{21}$ and localized modeling functions ${ }^{22}$ to address this problem, and also to make local model updates easier. However, these approaches require a procedure or analyst judgment for: 1) partitioning the explanatory variable space; 2) selecting the location of the local modeling functions in the explanatory variable space; and/or 3) selecting the mathematical structure of the local models.

In this work, the multivariate orthogonal function modeling first described in Ref. [20] was applied using both the explanatory variables and spline functions of the explanatory variables. This approach provides the required local nonlinear modeling capability, while retaining easy physical interpretation of the model, and automating the selection of optimal modeling complexity necessary to accurately characterize the functional dependencies.

The next sections describe the approach to global aerodynamic modeling using multivariate orthogonal functions derived from multivariate polynomials and spline functions of the measured explanatory variable data.

\section{A. Aircraft Aerodynamic Modeling}

For global aerodynamic modeling from flight data, nondimensional aerodynamic force and moment coefficients are used as the response variable (also called the dependent variable) in the modeling problem. A separate modeling problem is solved for each force or moment coefficient, corresponding to minimizing the equation error in each individual equation of motion for the six rigid-body degrees of freedom of the aircraft. Values for the nondimensional aerodynamic force and moment coefficients cannot be measured directly in flight, but instead must be computed from measured and known quantities using the following equations ${ }^{14}$

$$
\begin{gathered}
C_{X} \equiv-C_{A}=\frac{\left(m a_{x}-T_{x}\right)}{\bar{q} S} \quad C_{Y}=\frac{m a_{y}}{\bar{q} S} \quad C_{Z}=-C_{N}=\frac{\left(m a_{z}-T_{z}\right)}{\bar{q} S} \\
C_{D}=-C_{X} \cos \alpha-C_{Z} \sin \alpha \\
C_{l}=\frac{I_{x}}{\bar{q} S b}\left[\dot{p}-\frac{I_{x z}}{I_{x}}(p q+\dot{r})+\frac{\left(I_{z}-I_{y}\right)}{I_{x}} q r\right] \\
C_{m}=\frac{I_{y}}{\bar{q} S \bar{c}}\left[\dot{q}+\frac{\left(I_{x}-I_{z}\right)}{I_{y}} p r+\frac{I_{x z}}{I_{y}}\left(p^{2}-r^{2}\right)-M_{T}\right]
\end{gathered}
$$




$$
C_{n}=\frac{I_{z}}{\bar{q} S b}\left[\dot{r}-\frac{I_{x z}}{I_{z}}(\dot{p}-q r)+\frac{\left(I_{y}-I_{x}\right)}{I_{z}} p q\right]
$$

These expressions retain the full nonlinear dynamics in the aircraft equations of motion.

The result is $N$ values of the nondimensional force and moment coefficients, where $N$ is the number of data points. These values are often called measured force and moment coefficients, even though they not measured directly, but rather computed from other measurements and known quantities. Explanatory variables such as angle of attack, Mach number, pitch rate, and control surface deflections, are measured directly.

The desired form of the global aerodynamic model is a mathematical model structure with estimated model parameter values and associated uncertainties, relating the nondimensional aerodynamic force and moment coefficients to aircraft states and controls that can be measured.

\section{B. Multivariate Orthogonal Function Modeling}

The form of a multivariate orthogonal function model is

$$
z=a_{1} \boldsymbol{p}_{1}+a_{2} \boldsymbol{p}_{2}+\ldots+a_{n} \boldsymbol{p}_{n}+\boldsymbol{\varepsilon}
$$

where $z$ is an $N$-dimensional vector of the response variable (e.g., nondimensional force or moment coefficient), $z=\left[z_{1}, z_{2}, \ldots, z_{N}\right]^{T}$, modeled in terms of a linear combination of $n$ mutually orthogonal modeling functions $\boldsymbol{p}_{j}, j=1,2, \ldots, n$. Each $\boldsymbol{p}_{j}$ is an $N$-dimensional vector which in general depends on the explanatory variables. The $a_{j}, j=1,2, \ldots, n$ are constant model parameters to be determined, and $\boldsymbol{\varepsilon}$ denotes the modeling error vector.

Equation (9) represents a mathematical model used to represent functional dependencies in the measured data. The important questions of determining how the modeling functions $\boldsymbol{p}_{j}$ are computed from the explanatory variables, as well as which modeling functions should be included in Eq. (9), which implicitly determines $n$, will be addressed later. At this point, the properties of a multivariate orthogonal function model are examined.

Define an $N \times n$ matrix $\boldsymbol{P}$,

$$
\boldsymbol{P}=\left[\boldsymbol{p}_{1}, \boldsymbol{p}_{2}, \ldots, \boldsymbol{p}_{n}\right]
$$

and let $\boldsymbol{a}=\left[a_{1}, a_{2}, \ldots, a_{n}\right]^{T}$. Equation (9) can then be written as a standard least squares regression problem,

$$
z=\boldsymbol{P a}+\boldsymbol{\varepsilon}
$$

The error vector $\varepsilon$ is to be minimized in a least squares sense. The goal is to determine $\boldsymbol{a}$ that minimizes the least squares cost function

$$
J=\frac{1}{2}(z-P a)^{T}(z-P a)=\frac{1}{2} \varepsilon^{T} \varepsilon
$$

The parameter vector estimate $\hat{\boldsymbol{a}}$ that minimizes this cost function is computed as ${ }^{14}$

$$
\hat{\boldsymbol{a}}=\left[\boldsymbol{P}^{T} \boldsymbol{P}\right]^{-1} \boldsymbol{P}^{T} z
$$

The estimated parameter covariance matrix is ${ }^{14}$

$$
\boldsymbol{\Sigma}_{\hat{\boldsymbol{a}}}=E\left[(\hat{\boldsymbol{a}}-\boldsymbol{a})(\hat{\boldsymbol{a}}-\boldsymbol{a})^{T}\right]=\sigma^{2}\left(\boldsymbol{P}^{T} \boldsymbol{P}\right)^{-1}
$$


where $E$ is the expectation operator, and the fit error variance $\sigma^{2}$ can be estimated from the residuals

$$
v=z-P \hat{a}
$$

using

$$
\hat{\sigma}^{2}=\frac{1}{(N-n)}\left[(z-\boldsymbol{P} \hat{\boldsymbol{a}})^{T}(z-\boldsymbol{P} \hat{\boldsymbol{a}})\right]=\frac{\boldsymbol{v}^{T} \boldsymbol{v}}{(N-n)}
$$

Parameter standard errors are computed as the square root of the diagonal elements of the $\Sigma_{\hat{a}}$ matrix from Eq. (14), using $\hat{\sigma}^{2}$ from Eq. (16). The identified model output $\boldsymbol{y}$ is computed as

$$
\boldsymbol{y}=\boldsymbol{P} \hat{a}
$$

In conventional least squares modeling, the modeling functions (columns of $\boldsymbol{P}$ ) are often polynomials in the explanatory variables. This approach corresponds to using the terms of a multivariate Taylor series expansion to approximate the functional dependence of the response variable on the explanatory variables. If the modeling functions are instead multivariate orthogonal functions generated from the explanatory variable data, it is easier to determine an appropriate model structure, because the explanatory capability of each modeling function is completely distinct from all the others. This decouples the least squares modeling problem, as will be shown now.

For mutually orthogonal modeling functions,

$$
\boldsymbol{p}_{i}^{T} \boldsymbol{p}_{j}=0, i \neq j \quad, \quad i, j=1,2, \ldots, n
$$

and $\boldsymbol{P}^{T} \boldsymbol{P}$ is a diagonal matrix with the inner product of the orthogonal functions on the main diagonal. Using Eqs. (10) and (18) in Eq. (13), the $j^{\text {th }}$ element of the estimated parameter vector $\hat{\boldsymbol{a}}$ is given by

$$
\hat{a}_{j}=\left(\boldsymbol{p}_{j}^{T} z\right) /\left(\begin{array}{ll}
\boldsymbol{p}_{j}^{T} & \left.\boldsymbol{p}_{j}\right)
\end{array}\right.
$$

Using Eqs. (10), (18), and (19) in Eq. (12),

$$
\hat{J}=\frac{1}{2}\left[z^{T} z-\sum_{j=1}^{n}\left(\boldsymbol{p}_{j}^{T} z\right)^{2} /\left(\boldsymbol{p}_{j}^{T} \boldsymbol{p}_{j}\right)\right]
$$

Equation (20) shows that when the modeling functions are orthogonal, the reduction in the least square cost function resulting from including the term $a_{j} \boldsymbol{p}_{j}$ in the model depends only on the response variable data $z$ and the added orthogonal modeling function $\boldsymbol{p}_{j}$. The least squares modeling problem is therefore decoupled, which means each orthogonal modeling function can be evaluated independently in terms of its ability to reduce the least squares model fit to the data, regardless of which other orthogonal modeling functions are already selected for the model. When the modeling functions $\boldsymbol{p}_{j}$ are instead polynomials in the explanatory variables (or any other non-orthogonal function set), the least squares problem is coupled, and iterative analysis is required to find the subset of modeling functions for an adequate model structure.

The orthogonal modeling functions to be included in the model are chosen to minimize predicted squared error, $P S E$, defined by ${ }^{23}$

$$
P S E=\frac{(z-\boldsymbol{P} \hat{\boldsymbol{a}})^{T}(z-\boldsymbol{P} \hat{\boldsymbol{a}})}{N}+\sigma_{\max }^{2} \frac{n}{N}
$$




$$
P S E=\frac{2 \hat{J}}{N}+\sigma_{\max }^{2} \frac{n}{N}
$$

The constant $\sigma_{\max }^{2}$ is the upper-bound estimate of the squared error between future data and the model, i.e., the upper-bound mean squared error for prediction cases. The upper bound is used in the model over-fit penalty term to account for the fact that PSE is calculated when the model structure is not correct, i.e., during the model structure determination stage. Using the upper bound is conservative in the sense that model complexity will be minimized as a result of using an upper bound for this constant in the penalty term. Because of this, the value of PSE computed from Eq. (22) for a particular model structure tends to overestimate actual prediction errors on new data. Therefore, the PSE metric conservatively estimates the squared error for prediction cases.

A simple estimate of $\sigma_{\max }^{2}$ that is independent of the model structure can be obtained by considering $\sigma_{\max }^{2}$ to be the residual variance estimate for a constant model equal to the mean of the measured response values,

$$
\sigma_{\max }^{2}=\frac{1}{N-1} \sum_{i=1}^{N}\left[z_{i}-\bar{z}\right]^{2}
$$

where

$$
\bar{z}=\frac{1}{N} \sum_{i=1}^{N} z_{i}
$$

The PSE in Eq. (22) depends on the mean squared fit error, $2 \hat{J} / N$, and a term proportional to the number of terms in the model, $n$. The latter term prevents over-fitting the data with too many model terms, which is detrimental to model prediction accuracy ${ }^{4,14,23}$. While the mean squared fit error $2 \hat{J} / N$ must decrease with the addition of each orthogonal modeling function to the model (by Eq. (20)), the over-fit penalty term $\sigma_{\max }^{2} n / N$ must increase with each added model term ( $n$ increases). Introducing the orthogonal modeling functions into the model in order of most effective to least effective in reducing the mean squared fit error (quantified by $\left(\boldsymbol{p}_{j}^{T} z\right)^{2} /\left(\boldsymbol{p}_{j}^{T} \boldsymbol{p}_{j}\right)$ for the $j^{\text {th }}$ orthogonal modeling function) means that the PSE metric will always have a single global minimum.

Figure 5 depicts this graphically, using actual modeling results from Ref. [4]. The figure shows that after the first 6 modeling functions, the added model complexity associated with an additional orthogonal modeling function is not justified by the associated reduction in mean squared fit error. This point is marked by a minimum $P S E$, which defines an adequate model structure with good predictive capability. Ref. [23] contains further statistical arguments and analysis for the form of PSE given in Eq. (22), including justification for its use in modeling problems.

Using orthogonal functions to model the response variable makes it possible to evaluate the merit of including each modeling function individually, using the predicted squared error PSE. The goal is to select a model structure with minimum PSE, and the PSE always has a single global minimum for orthogonal modeling functions. This makes the model structure determination a well-defined and straightforward process that can be (and was) automated.

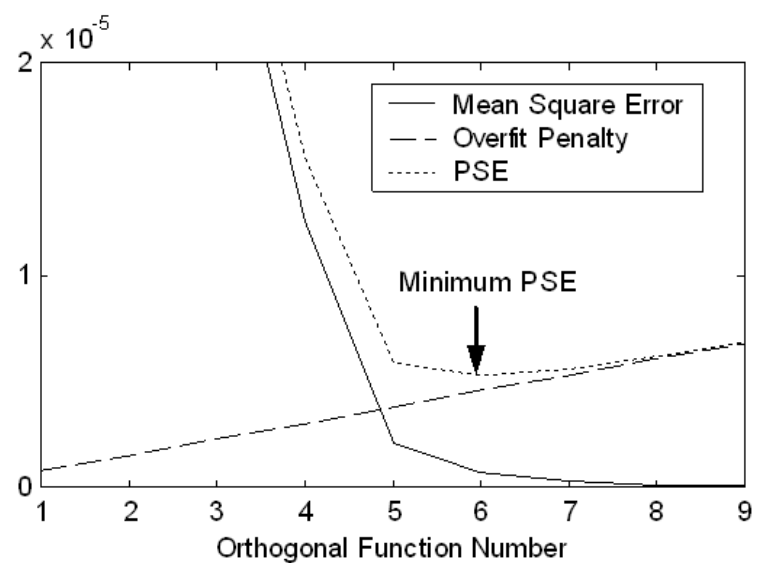

Figure 5. Model structure determination using orthogonal functions and PSE 


\section{Generating Orthogonal Modeling Functions}

Multivariate orthogonal functions can be generated from ordinary multivariate functions in the explanatory variables using a Gram-Schmidt orthogonalization procedure. This approach is described in Refs. [4] and [14], which are the basis for the material presented here.

The process begins by choosing one of the ordinary multivariate functions as the first orthogonal function. Typically, a vector of ones (associated with the bias term in the model) is chosen as the first orthogonal function,

$$
p_{1}=1
$$

In general, any function of the explanatory variables can be chosen as the first orthogonal function, without any change in the procedure. To generate the next orthogonal function, an ordinary multivariate polynomial function is made orthogonal to the preceding orthogonal function(s). Define the $j^{\text {th }}$ orthogonal function $\boldsymbol{p}_{j}$ as

$$
\boldsymbol{p}_{j}=\boldsymbol{\xi}_{j}-\sum_{k=1}^{j-1} \gamma_{k j} \boldsymbol{p}_{k} \quad j=2,3, \ldots, n_{t}
$$

where $\xi_{j}$ is the $j^{\text {th }}$ ordinary multivariate function vector. For example, each $\xi_{j}$ could be some ordinary polynomial function of the explanatory variables or a spline function of the explanatory variables. The $\gamma_{k j}$ for $k=1,2, \ldots, j-1$ are scalars determined by multiplying both sides of Eq. (26) by $\boldsymbol{p}_{k}^{T}$, then invoking the mutual orthogonality of the $\boldsymbol{p}_{k}, k=1,2, \ldots, j$, and solving for $\gamma_{k j}$

$$
\gamma_{k j}=\frac{\boldsymbol{p}_{k}^{T} \boldsymbol{\xi}_{j}}{\boldsymbol{p}_{k}^{T} \boldsymbol{p}_{k}} \quad k=1,2, \ldots, j-1
$$

The same process can be implemented in sequence for each ordinary multivariate function $\xi_{j}, j=2,3, \ldots, n_{t}$. The total number of ordinary multivariate functions used as raw material for generating the multivariate orthogonal functions, including the bias term, is $n_{t}$. It can be seen from Eqs. (25)-(27) that each orthogonal function can be expressed exactly in terms of a linear expansion of the original multivariate functions. The orthogonal functions are generated sequentially by orthogonalizing the original multivariate functions with respect to the orthogonal functions already computed, so that each orthogonal function can be considered an orthogonalized version of an original multivariate function.

The multivariate orthogonal function generation method described here normally starts by generating all possible ordinary multivariate polynomials in the explanatory variables, up to a selected maximum order. For example, when modeling the nondimensional vertical force coefficient $C_{Z}$, the explanatory variables might be angle of attack $\alpha$, nondimensional pitch rate $\hat{q} \equiv q \bar{c} / 2 V$, and elevator deflection $\delta_{e}$. If the selected maximum order is 3 , then the ordinary multivariate polynomial modeling functions used as the raw material for the orthogonalization process would include terms like $\alpha, \hat{q}, \delta_{e}, \alpha^{2}, \delta_{e}^{3}, \alpha \hat{q}^{2}, a \hat{q} \delta_{e}$, etc. Note that considering any other candidate explanatory variables, such as sideslip angle $\beta$, can be done by simply including the sideslip angle among the group of explanatory variables. If it turns out that the sideslip angle is not needed to model $C_{Z}$, the model structure determination using orthogonal modeling functions will not select any orthogonal functions associated with sideslip angle. This occurs naturally and automatically in the course of the model structure determination process described earlier. Therefore, there is no harm in including explanatory variables that might not be important, except that additional computation time will be required to identify the model structure, because additional multivariate orthogonal functions will be generated and sorted. Similarly, if the maximum order is chosen higher than necessary, the only penalty would be the increased computation time necessary for generating and sorting the additional orthogonal functions. The final identified model would be the same. Consequently, the choices that the analyst needs to make are very easy and not critical to the quality of the final results. 
If the $\boldsymbol{p}_{j}$ vectors and the $\boldsymbol{\xi}_{j}$ vectors are arranged as columns of matrices $\boldsymbol{P}$ and $\boldsymbol{X}$, respectively, and the $\gamma_{k j}$ are elements in the $k^{\text {th }}$ row and $j^{\text {th }}$ column of an upper triangular matrix $\boldsymbol{G}$ with ones on the diagonal,

$$
\boldsymbol{G}=\left[\begin{array}{ccccc}
1 & \gamma_{12} & \gamma_{13} & \cdots & \gamma_{1 n_{t}} \\
0 & 1 & \gamma_{23} & \cdots & \gamma_{2 n_{t}} \\
0 & 0 & 1 & \cdots & \gamma_{3 n_{t}} \\
\vdots & \vdots & \vdots & \vdots & \vdots \\
0 & 0 & 0 & \cdots & 1
\end{array}\right]
$$

Then

$$
\boldsymbol{X}=\boldsymbol{P} \boldsymbol{G}
$$

which leads to

$$
\boldsymbol{P}=\boldsymbol{X} \boldsymbol{G}^{-1}
$$

The columns of $\boldsymbol{G}^{-1}$ contain the coefficients for expansion of each column of $\boldsymbol{P}$ (i.e., each multivariate orthogonal function) in terms of an exact linear expansion in the original multivariate functions in the columns of $\boldsymbol{X}$. Equation (30) can be used to express each multivariate orthogonal function in terms of the original multivariate functions. The manner in which the orthogonal functions are generated allows them to be decomposed without ambiguity into an expansion of the original multivariate functions, which have physical meaning.

\section{Conversion to Physically-Meaningful Multivariate Function Models}

After the model structure is determined using multivariate orthogonal modeling functions for minimum PSE, the identified model output is

$$
\boldsymbol{y}=\boldsymbol{P} \hat{\boldsymbol{a}}
$$

where the $\boldsymbol{P}$ matrix now includes only the $n$ orthogonal functions selected in the model structure determination, $n \leq n_{t}$. Each retained orthogonal modeling function can be decomposed without error into an expansion of the original multivariate functions in the explanatory variables, using the columns of $\boldsymbol{G}^{-1}$ in Eq. (28) corresponding to the retained orthogonal functions. Common terms are combined using double precision arithmetic to arrive finally at a model using only original multivariate functions in the explanatory variables. Terms that contribute less than 0.1 percent of the final model root-mean-square magnitude are dropped.

The final form of the model is a sum of ordinary multivariate functions in the explanatory variables, with associated model parameter estimates. Examples of the final model forms obtained are given later in the Results sections.

\section{E. Including Spline Functions in the Orthogonalization}

For global aerodynamic modeling, functional dependencies of the aerodynamic coefficients on the explanatory variables can exhibit significant localized variation. In these cases, a global polynomial model can be inadequate for capturing those local variations. To solve this problem, spline functions in the explanatory variables can be introduced as additional pseudo-explanatory variables. Splines have local modeling capability that global polynomial modeling functions do not have, because splines are polynomials defined only on selected intervals. Low-order polynomial terms defined on limited intervals can approximate nonlinearities quite well. Using polynomial splines also retains clear physical interpretation in the final model.

Spline functions are defined as piecewise polynomials functions of degree $m$ in one or more explanatory variables. The term "piecewise" means that the polynomial is different for specific ranges of the explanatory variables. Spline function values and derivatives agree at the points where the piecewise polynomials join. These 
points are called knots, and are defined as specific values of each explanatory variable. A polynomial spline $S_{m}(x)$ of degree $m$ with continuous derivatives up to degree $m-1$, for a single explanatory variable $x \in\left[x_{0}, x_{\max }\right]$, can be expressed as

$$
S_{m}(x)=\sum_{r=1}^{m} C_{r} x^{r}+\sum_{i=1}^{k} D_{i}\left(x-x_{i}\right)_{+}^{m}
$$

where

$$
\left(x-x_{i}\right)_{+}^{m}=\left\{\begin{array}{cc}
\left(x-x_{i}\right)^{m} & x>x_{i} \\
0 & x \leq x_{i}
\end{array}\right.
$$

and the $C_{r}$ and $D_{i}$ are constants and $\left(x-x_{i}\right)_{+}^{m}$ are the piecewise parts of the spline function. The values $x_{1}, x_{2}, \ldots, x_{k}$ are knots which satisfy the condition

$$
x_{0}<x_{1}<x_{2}<\ldots<x_{k}<x_{\max }
$$

Three piecewise spline types are sketched in Figure 6 for the same selected knots in angle of attack.

Note that when the spline knots are the same, a higher-order piecewise spline in a single explanatory variable can be computed as a multiplication of lower-order piecewise splines in the same explanatory variable,

$$
\left(x-x_{i}\right)_{+}^{m}=\left(x-x_{i}\right)_{+}^{m-1}\left(x-x_{i}\right)_{+}^{1} \quad m \geq 1
$$

This fact can be used advantageously in generating multivariate orthogonal modeling functions with excellent local modeling capability. This is best demonstrated with a simple example.

Suppose that the nondimensional vertical aerodynamic force coefficient $C_{Z}$ is being modeled with explanatory variables angle of attack $\alpha$ and elevator control deflection $\delta_{e}$. For a maximum selected model order 2, the ordinary polynomial modeling functions that will serve as raw material for the orthogonalization process are:

$$
1, \alpha, \alpha^{2}, \alpha \delta_{e}, \delta_{e}, \delta_{e}^{2}
$$

which would lead to a final multivariate polynomial model of the form

$$
C_{Z}=C_{Z_{o}}+C_{Z_{\alpha}} \alpha+C_{Z_{\alpha^{2}}} \alpha^{2}+C_{Z_{\alpha \delta_{e}}} \alpha \delta_{e}+C_{Z_{\delta_{e}}} \delta_{e}+C_{Z_{\delta_{e}^{2}}} \delta_{e}^{2}
$$

where the values of the model parameters such as $C_{Z_{o}}$ and $C_{Z_{\alpha \delta_{e}}}$ would be estimated from the data using the orthogonal function modeling and subsequent decomposition procedures described earlier, and some of the terms might not be present, depending on the results of the model structure determination using orthogonal functions. This represents a global polynomial model.

Now introduce a first-order spline term in angle of attack with a single knot located at $10 \mathrm{deg}$. Using the same maximum model order 2 , the set of ordinary polynomial modeling functions expands to

$$
1, \alpha, \alpha^{2}, \alpha \delta_{e}, \delta_{e}, \delta_{e}^{2},(\alpha-10)_{+}^{1}, \alpha(\alpha-10)_{+}^{1}, \delta_{e}(\alpha-10)_{+}^{1},(\alpha-10)_{+}^{2}
$$

which would lead to a final multivariate polynomial model of the form

$$
\begin{aligned}
C_{Z}= & C_{Z_{o}}+C_{Z_{\alpha}} \alpha+C_{Z_{\alpha^{2}}} \alpha^{2}+C_{Z_{\alpha \delta_{e}}} \alpha \delta_{e}+C_{Z_{\delta_{e}}} \delta_{e}+C_{Z_{\delta_{e}^{2}}} \delta_{e}^{2} \\
& +C_{Z_{\alpha_{10}^{1}}}(\alpha-10)_{+}^{1}+C_{Z_{\alpha \alpha_{10}^{1}}} \alpha(\alpha-10)_{+}^{1}+C_{Z_{\delta_{e} \alpha_{10}^{1}}} \delta_{e}(\alpha-10)_{+}^{1}+C_{Z_{\alpha_{10}^{2}}}(\alpha-10)_{+}^{2}
\end{aligned}
$$


As before, some of the terms in Eq. (39) might be not be present, depending on the results of the model structure determination using orthogonal functions.
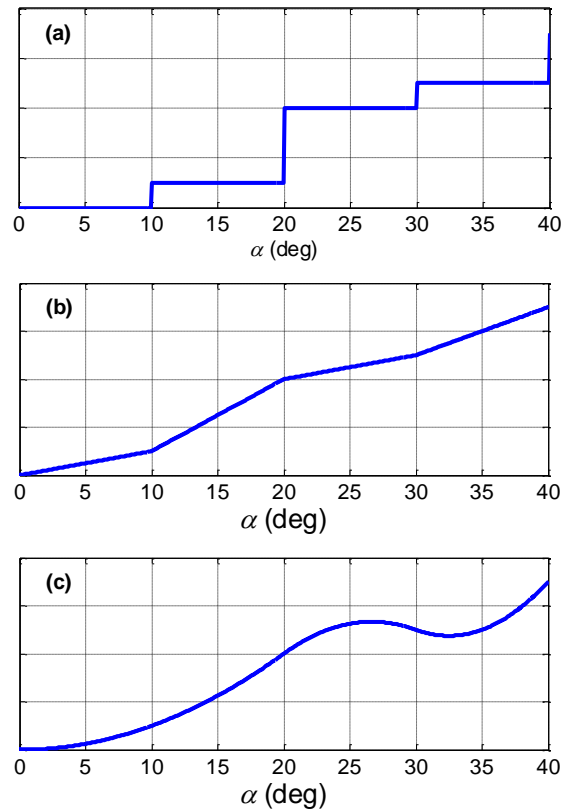

Figure 6. Polynomial splines in angle of attack:

(a) zero-degree, (b) first-degree, (c) second-degree

With this expanded set of ordinary polynomial modeling functions, more orthogonal functions will be generated, and there is now capability to accommodate changes in the linear dependence of $C_{Z}$ on $\alpha$ for $\alpha>10 \mathrm{deg}$, as well as additional $\alpha^{2}$ and $\alpha \delta_{e}$ nonlinearities that only take effect when $\alpha>10 \mathrm{deg}$. Note also that $\alpha(\alpha-10)_{+}^{1}$ and $(\alpha-10)_{+}^{2}$, for example, are in general not the same function. Because higherorder splines are created automatically by the multiplication of first-order splines (cf. Eq. (35)), only first-order splines in the explanatory variables need to be included as pseudo-explanatory variables.

Extrapolating from this simple example, it is clear that multiple knots in the explanatory variables would provide a very effective nonlinear modeling capability in multiple dimensions with local nonlinear modeling capability, while retaining physical insight into the functional dependencies. The automated orthogonalization and sorting process described earlier identifies which nonlinear terms are necessary to characterize the functional dependencies and estimates the associated model parameters. Inputs required from the analyst relate only to the limits of what should be considered, such as which explanatory variables to consider, maximum model order to consider, and knot locations to consider. However, these can be specified very generously, because the orthogonal function modeling algorithm automatically sorts out which of the terms are important, based on the data, and discards the rest. The result is a global parsimonious model with excellent local nonlinear modeling capability and easy physical interpretation.

\section{Test Aircraft and Flight Data}

\section{A. T-2 Subscale Jet Transport Aircraft Description}

The T-2 aircraft is a 5.5 percent dynamically-scaled model of a generic commercial twin-engine jet transport aircraft. Figure 7 shows a photograph of the aircraft in flight. The aircraft has twin jet engines mounted under the wings and retractable tricycle landing gear. Aircraft geometry and nominal mass properties are given in Table 3 . Further information on the T-2 subscale jet transport aircraft and associated flight test operations can be found in Refs. [24]-[26]. A similar airframe was tested extensively in the wind tunnel ${ }^{8}$, although the wind tunnel test article differed in some geometric details from the aircraft used for the flight tests. The wind tunnel data provided a reference for comparison with the aerodynamic models identified directly from flight data.

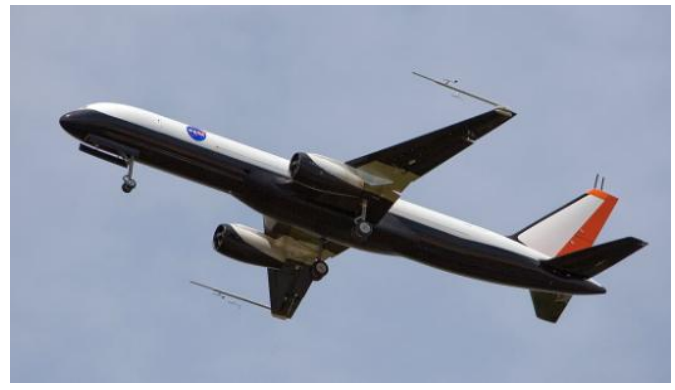

Figure 7. T-2 subscale jet transport aircraft Credit: NASA Langley Research Center

\section{Control Surfaces}

Control surfaces on the T-2 aircraft are left and right ailerons, left and right inboard and outboard elevators, upper and lower rudders, left and right inboard and outboard trailing-edge flaps, and left and right inboard and outboard spoilers, for a total of 16 independent control surfaces. For the flight data analyzed in this work, only the elevators, ailerons, and rudders were deflected. The individual elevator surfaces were moved together as a single elevator surface, and similarly for the rudders. Left and right ailerons were deflected asymmetrically, in the 
conventional way. Definitions of control surface deflections are given below. Trailing edge down is positive deflection for wing and elevator surfaces, and trailing edge left is positive for rudder surfaces.

$$
\begin{gathered}
\delta_{e}=\frac{1}{4}\left(\delta_{e_{l o}}+\delta_{e_{l i}}+\delta_{e_{r i}}+\delta_{e_{r o}}\right) \\
\delta_{a}=\frac{1}{2}\left(\delta_{a_{r}}-\delta_{a_{l}}\right) \quad \delta_{r}=\frac{1}{2}\left(\delta_{r_{u}}+\delta_{r_{l}}\right)
\end{gathered}
$$

The aircraft can be flown by a safety pilot using direct visual contact and conventional radio control. A research pilot executed the flight test maneuvers from inside a mobile control room, using a synthetic vision display drawn from telemetry data and a local terrain database, along with video from a camera in the nose of the aircraft. Inputs from the research pilot and a ground-based flight control system were used to generate control surface commands which were transmitted by telemetry to the aircraft.

The flight control system has the capability to inject automated control surface perturbations to excite the aircraft dynamic response for modeling purposes. These control surface perturbations can have arbitrary waveforms, and can be applied to multiple control surfaces individually or simultaneously. The perturbations are summed with pilot and feedback control commands in the flight control system, just before the actuator command rate and position limiting. Typically, the research pilot flies the aircraft to the desired flight condition(s), then initiates the automated control surface perturbations with a trigger switch on the control stick.

\section{Instrumentation and Data Acquisition}

The T-2 aircraft was equipped with a micro-INS, which provided 3-axis translational accelerometer measurements, angular rate measurements, estimated attitude angles, and GPS velocity and position. Air data probes attached to booms mounted on each wingtip (visible in Figure 7) measured angle of attack, sideslip angle, static pressure, and dynamic pressure. Measurements from static pressure sensors and ambient temperature sensors were used to compute air density and altitude. Engine speeds in rpm were measured and used as inputs to an engine model to compute thrust. The engine model was identified from ground test data, with adjustments for ram drag identified from flight data. Potentiometers on the rotation axes of the control surfaces measured control surface deflections. Mass properties were computed based on measured fuel flow, pre-flight weight and balance, and inertia measurements done on the ground for the aircraft without fuel. The pilot stick and rudder pedal commands and throttle position were also measured and recorded. Data from onboard sensors were telemetered to the ground in real time. Sampling rate for the flight data was $200 \mathrm{~Hz}$, decimated to $50 \mathrm{~Hz}$ for data analysis and modeling.

\section{Simulation Results}

The techniques for flight test maneuver design and global aerodynamic modeling were applied to a nonlinear aircraft simulation of the T-2 subscale jet transport aircraft. The simulation was based on wind tunnel data ${ }^{8}$ collected using a wind tunnel model with geometry similar to the T-2 aircraft. Full nonlinear equations of motion were implemented and solved in MATLAB ${ }^{\circledR}$ using aircraft simulation software included in SIDPAC ${ }^{14}$, adapted for the T-2 aircraft. Documentation for the nonlinear aircraft simulation software can be found in Appendix D of Ref. [14]. The engine model was identified from ground test data, with ram drag corrections identified from T-2 flight data. Simulated aircraft responses were corrupted with white Gaussian noise using root-mean-square amplitudes similar to what was observed in the T-2 flight data.

Figure 8 shows control surface deflections and aircraft responses for a maneuver with orthogonal optimized multi-sine perturbation inputs applied to the elevator, aileron, and rudder control surfaces simultaneously, while the pilot implemented a slow increase in angle of attack by gradually pulling aft on the longitudinal stick with constant power setting. This is the flight test maneuver described in Section II for collecting data for global aerodynamic modeling. Figure 9 shows a cross-plot of angle of attack and sideslip angle, demonstrating how the angle of attack and sideslip angle are excited in an uncorrelated fashion, similar to Figure 4. Analogous cross-plots could be made for the other aircraft responses and control surface deflections. 
Applying the global modeling techniques in simulation allows comparison of the global aerodynamic model identified from the simulated data with the known underlying aerodynamic database for the simulation. The aerodynamic database in the simulation was implemented in tabular form using wind tunnel data.
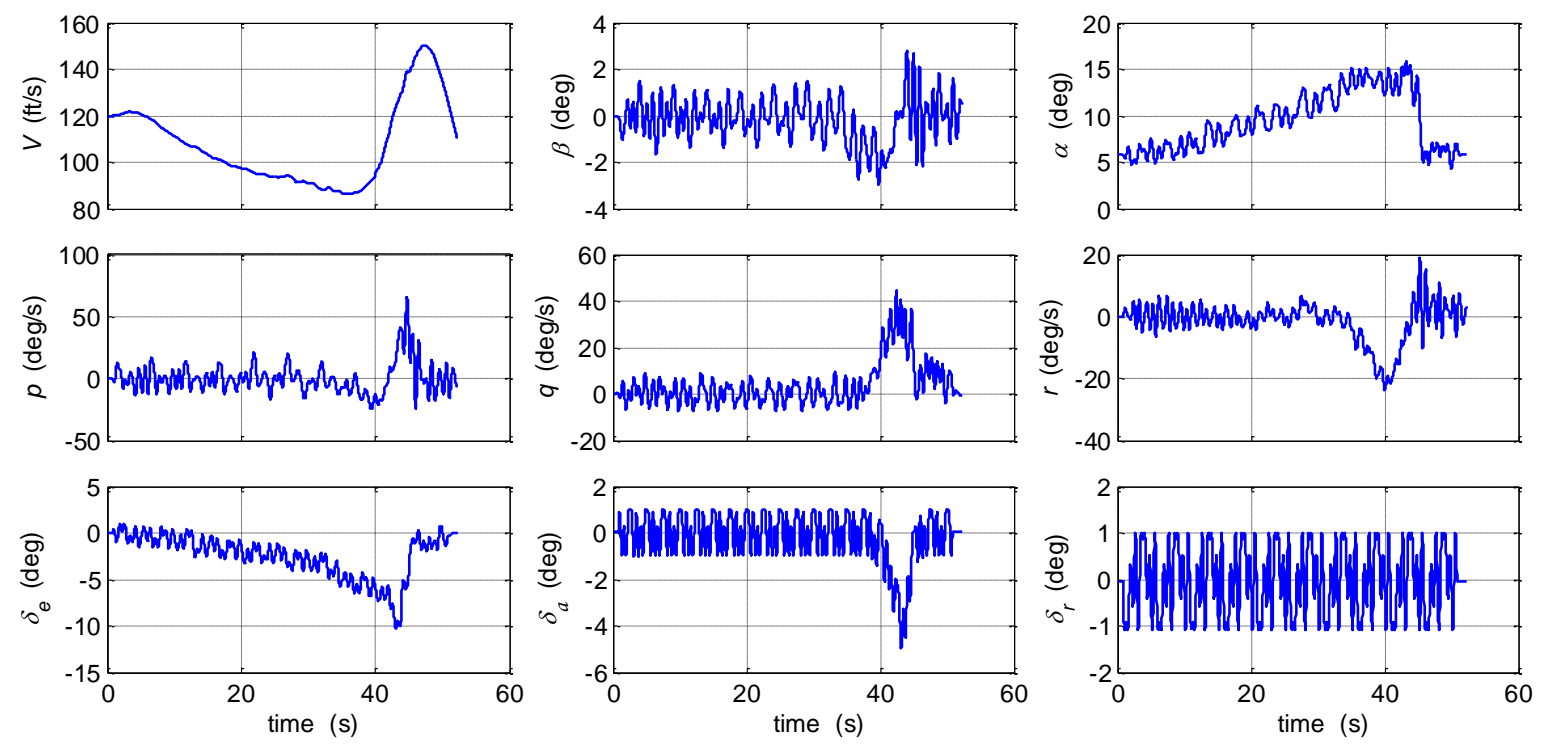

Figure 8. Simulated flight data for a global aerodynamic modeling maneuver

For the global aerodynamic modeling, initial modeling was done using coarse spacing of the knot locations in each explanatory variable to identify which explanatory variables required the increased local nonlinear modeling capability provided by spline terms. This analysis showed that the global modeling problem for the T-2 simulation data shown in Figure 8 required spline terms only for angle of attack. First-order splines in angle of attack with

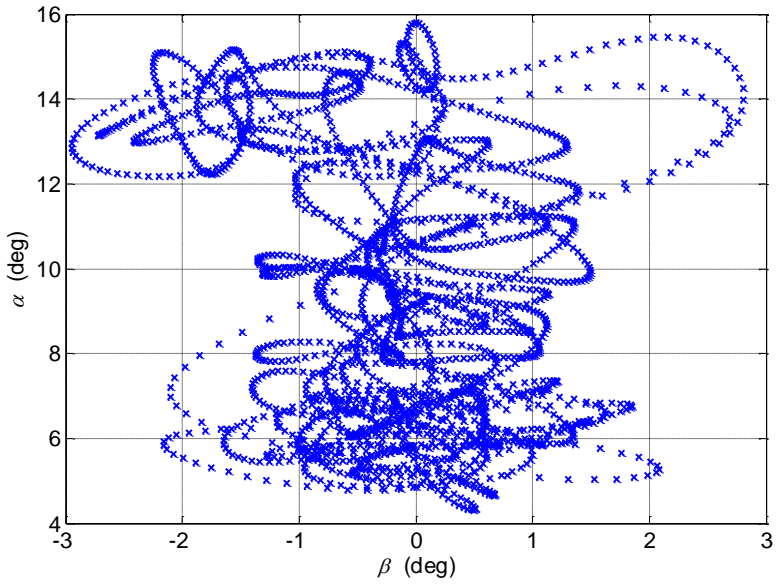

Figure 9. Simulated flight data cross plot for a global aerodynamic modeling maneuver knots finely spaced at $6,8,10,12$, and 14 deg were added as pseudo-explanatory variables for the global aerodynamic modeling. The spline knot spacing could be made finer, with no penalty other than increased computation time to identify the model structure, as discussed earlier. For each nondimensional force and moment coefficient model, model terms with up to third-order complexity were orthogonalized and sorted to identify an adequate model structure and estimate the associated model parameters values and uncertainties. The third-order complexity applied to the conventional explanatory variables (such as angle of attack, nondimensional pitch rate, and elevator control deflection), and also to the first-order splines in angle of attack, which were included as pseudo-explanatory variables. Consequently, the first-order spline terms were also multiplied by themselves and each other, up to third order, so that this approach really allowed for up to third-order spline terms in the modeling.

Figure 10 shows three-dimensional plots comparing the simulated flight data ( $\mathrm{x}$ markers) with a mesh surface drawn by interrogating the aerodynamic database in the simulation and a smooth surface drawn using the global aerodynamic model identified from simulated flight data alone. The ranges of the explanatory variables used to generate the mesh surface and the smooth surface were chosen to include all values from the simulated flight data. The results in Figure 10 show that for all force and moment coefficients, the smooth surface and mesh surface are very similar, indicating that the global aerodynamic modeling approach captured the underlying functional 
dependencies accurately and efficiently, using data from a single flight test maneuver. This is true even in cases with complex local nonlinearity, as in the case of axial force coefficient $C_{X}$ and rolling moment coefficient $C_{l}$.
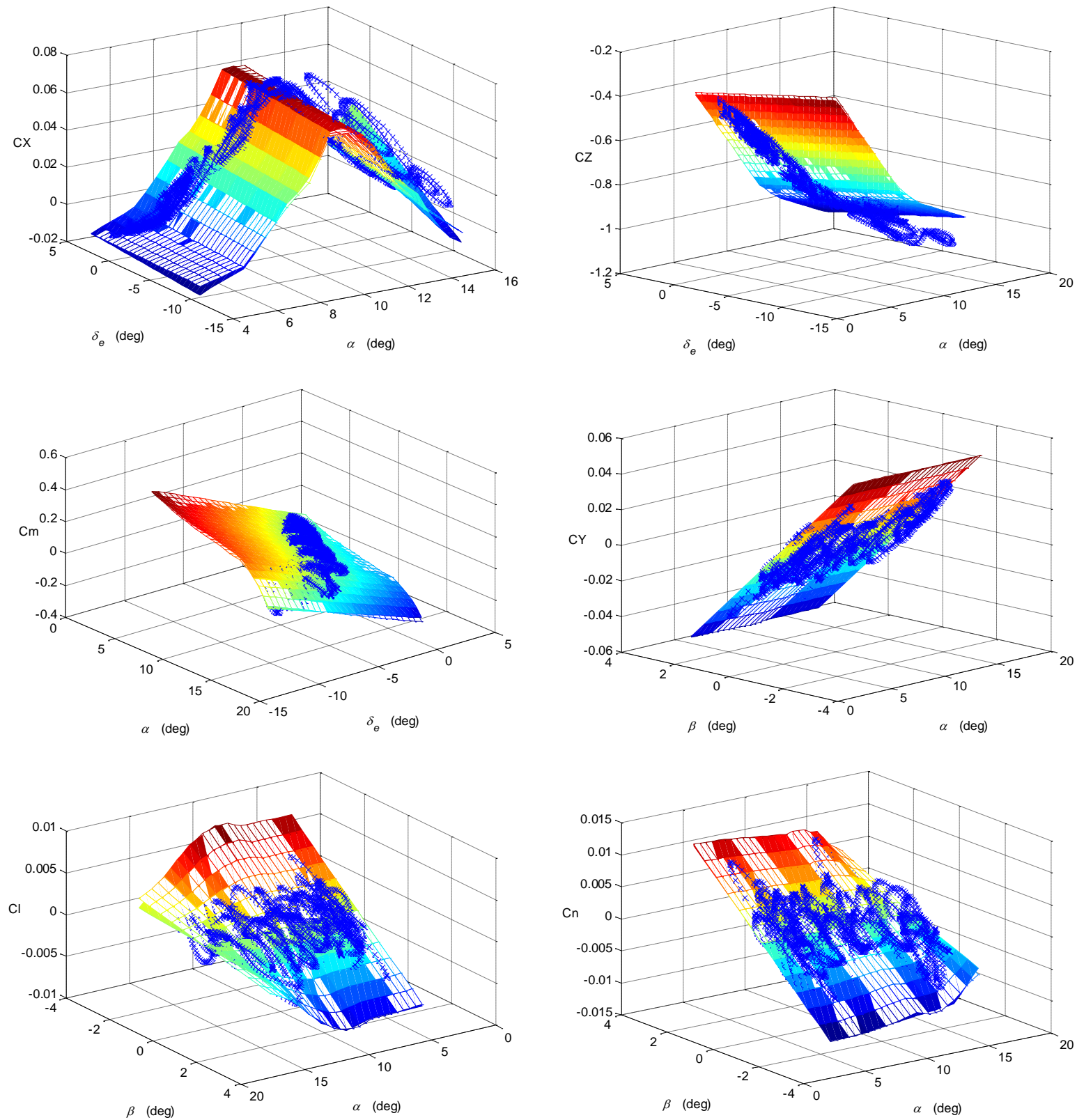

Figure 10. Global aerodynamic models identified from simulated flight data

Close inspection shows only small mismatches between the smooth surface and mesh surface at locations relatively far from the simulated flight data, which would be areas of extrapolation for the identified global model. Flying another maneuver through that part of the explanatory variable subspace would provide the data necessary to correct those small mismatches. The three-dimensional plots shown in Figure 10 must necessarily omit some of the explanatory variables (because there are more than two explanatory variables, and the third dimension is used for the response variable), so the mismatch between simulated flight data and the smooth and mesh surfaces is mostly a dependence on an explanatory variable that cannot be shown in the three-dimensional plot. The results in Figure 10 show that a few well-flown maneuvers of the type described here could very effectively cover a large portion of the 
explanatory variable subspace and therefore could be used in place of wind tunnel testing that requires extensive grids of test points to generate the aerodynamic database. The approach described here could be extended to more than the three control surfaces used in this demonstration ${ }^{16}$, further increasing the productivity and efficiency of the flight test maneuver and the global aerodynamic modeling process.

As an example of the final global modeling results, the global model identified for nondimensional aerodynamic vertical force coefficient $C_{Z}$ from simulated flight data was

$$
C_{Z}=C_{Z_{o}}+C_{Z_{\alpha}} \alpha+C_{Z_{q}} \frac{q \bar{c}}{2 V}+C_{Z_{\delta_{e}}} \delta_{e}+C_{Z_{\alpha_{10}^{1}}}(\alpha-10)_{+}^{1}+C_{Z_{\alpha_{12}^{1}}}(\alpha-12)_{+}^{1}
$$

where model parameter estimates and uncertainties are given in Table 4.

\section{Flight Test Results}

Flight test maneuvers similar to the one used for the simulation demonstration were flown on the T-2 subscale jet transport aircraft to collect data for global aerodynamic modeling. Figure 3 shows measured control surface deflections and aircraft responses for one of these maneuvers, with orthogonal optimized multi-sine perturbation inputs applied to the elevator, aileron, and rudder control surfaces simultaneously, while the pilot implemented a slow increase in angle of attack by gradually pulling aft on the longitudinal stick at constant idle power setting. The pilot also applied relatively large longitudinal and lateral stick inputs at roughly $20 \mathrm{sec}$, to recover the aircraft from a roll-off departure that occurred near 14 deg angle of attack. Automated perturbation inputs continued throughout the departure and recovery.
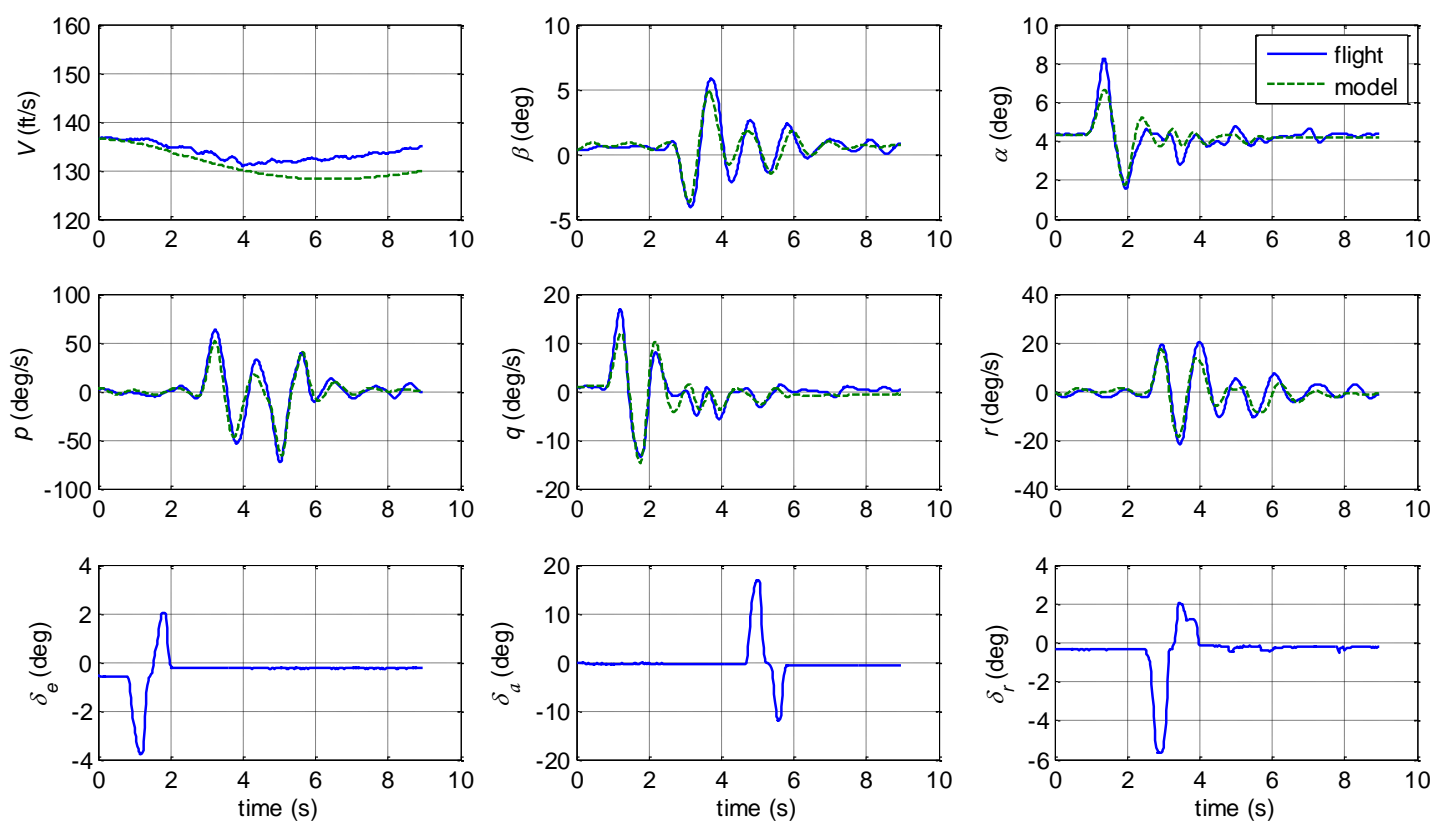

Figure 11. Prediction using global aerodynamic models identified from T-2 flight data

Detailed specifications of global aerodynamic models identified from flight data alone for all six nondimensional aerodynamic force and moment coefficients are given in Tables 5 and 6 . These models were identified using the global aerodynamic modeling procedure applied to flight data from only the single maneuver shown in Figure 3. The identified global aerodynamic models were then installed in the nonlinear aircraft simulation for prediction testing.

Figure 11 shows a prediction test using flight data from a piloted doublet sequence on longitudinal stick, rudder pedal, and lateral stick. The flight data used for this prediction test was of course not used in identifying the global 
aerodynamic models. The solid lines show flight data and the dashed lines were computed by applying measured control surface deflections from the flight data to the nonlinear aircraft simulation, using the global aerodynamic models from Tables 5 and 6 for the aerodynamics. The results show reasonable accuracy in predicting the aircraft response for this maneuver, which would be classified as a local maneuver. This maneuver was chosen because it involved localized multi-axis aircraft response to elevator, rudder, and aileron deflections dissimilar from the inputs used to identify the global aerodynamic model. The predictions show that the global aerodynamic modeling approach applied to flight data from only a single flight test maneuver produced a reasonable prediction of the flight responses, indicating that the functional dependencies of the nondimensional force and moment coefficients on the explanatory variables were captured by the global aerodynamic modeling procedure. Presumably, using flight data from multiple runs of global aerodynamic modeling maneuvers similar to the one shown in Figure 3 would produce even better global aerodynamic modeling results. This is currently being investigated. In addition, it should be possible to identify thrust effects using global aerodynamic modeling maneuvers at different power settings, such as those listed in Table 2.
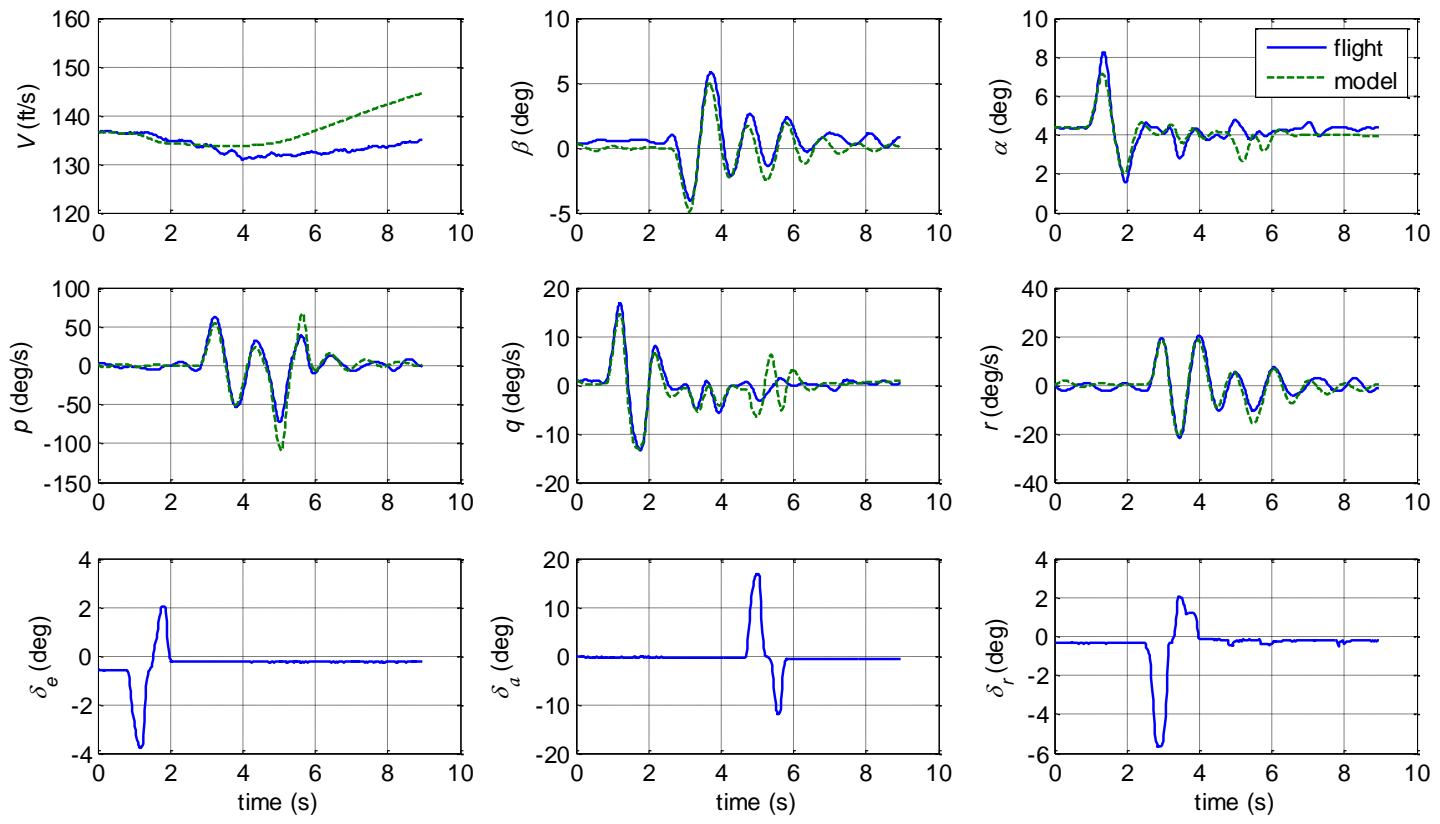

Figure 12. Prediction using T-2 wind tunnel database

Figure 12 shows prediction results for the same piloted doublet sequence maneuver, but instead using the wind tunnel database ${ }^{8}$ for the aerodynamics in the nonlinear aircraft simulation. Figures 11 and 12 show similar prediction accuracy, suggesting that the global aerodynamic modeling procedure applied to data from a single flight test maneuver can produce simulation results with accuracy comparable to what can be obtained using aerodynamic data from an extensive wind tunnel test. In fact, the root-mean-square of the differences between the flight data and the predictions using the identified global model, shown in Figure 11, was 20 percent lower than for the predictions using the wind tunnel database, shown in Figure 12. Finally, the compact analytical form of the global aerodynamic model provides significant insight into the functional dependencies and is compact enough to be shown in Tables 5 and 6. 


\section{Concluding Remarks}

Simulation and flight test data for a subscale jet transport aircraft were used to demonstrate a novel technique for efficiently and effectively identifying global aerodynamic models from a single flight test maneuver. Novel flight test maneuver design, an orthogonal function modeling technique using splines, and an automated modeling process were combined to create an approach that very efficiently produced accurate global aerodynamic models with easy physical interpretation, based on flight data alone. The resulting global aerodynamic models were incorporated into a nonlinear simulation that exhibited reasonable prediction capability for data from flight maneuvers that were not used in the modeling process.

The global modeling flight test maneuver was used to collect data over relatively large ranges of the explanatory variables for all six rigid-body degrees of freedom of the aircraft simultaneously. This resulted in highly efficient collection of flight data with de-correlated explanatory variables, for good model identifiability and modeling accuracy. The global aerodynamic modeling method incorporated spline functions in an automated orthogonal function modeling scheme, to allow accurate modeling of local complicated functional dependencies without adverse effects on other parts of the global model.

Accurate global models identified from flight data alone can be used in rapid and relatively low-budget unmanned aircraft programs to save development time and money by making it possible to generate an accurate nonlinear aircraft simulation from a single flight, without the need for extensive wind tunnel testing or aerodynamic calculations. The capability also has important implications for aircraft safety, because the technique could be used to generate an onboard model of the global aerodynamics for an aircraft. The approach could be used to account for the particular geometry or flight environment of an individual aircraft, as well as provide a capability to monitor and account for changes in the aircraft due to failures, damage, and airframe icing, for example. A global aerodynamic model identified from flight data alone could also be compared to a simulation database generated from wind tunnel data and aerodynamic calculations for the purpose of improving the fidelity of these ground-based aerodynamic prediction methods. This could be done through identifying where there are significant differences between the database generated using ground-based methods and the global model identified from flight data alone, as well as where the models agreed well. Such comparisons could also be used to estimate full-scale Reynolds number effects, or artifacts of wind tunnel testing, such as sting interference and wall effects.

\section{Acknowledgments}

The efforts of the AirSTAR flight test team at NASA Langley in building and testing the T-2 aircraft and associated systems, carefully calibrating the instrumentation, and carrying out the flight operations to collect the high-quality flight data used in this study, are gratefully acknowledged. Research in aircraft system identification is funded by the NASA Aviation Safety Program, Vehicle Systems Safety Technologies (VSST) project.

\section{References}

${ }^{1}$ Cunningham, K., Foster, J.V., Morelli, E.A., and Murch, A.M., "Practical Application of a Subscale Transport Aircraft for Flight Research in Control Upset and Failure Conditions," AIAA-2008-6200, AIAA Atmospheric Flight Mechanics Conference, Honolulu, HI, August 2008.

${ }^{2}$ Stevens, B.L. and Lewis, F.L., Aircraft Control and Simulation, John Wiley \& Sons, Inc., New York, NY, 1992.

${ }^{3}$ Cunningham, K., Foster, J.V., Shah, G.H., Stewart, E.C., Rivers, R.A., Wilborn, J.E., and Gato, W., "Simulation Study of a Commercial Transport Airplane During Stall and Post-Stall Flight", SAE 2004-01-3100, 2004 SAE World Aviation Congress \& Exposition, Reno, NV, November 2004.

${ }^{4}$ Morelli, E.A. and DeLoach, R., "Wind Tunnel Database Development using Modern Experiment Design and Multivariate Orthogonal Functions,” AIAA Paper 2003-0653, 41 ${ }^{\text {st }}$ AIAA Aerospace Sciences Meeting and Exhibit, Reno, NV, January 2003.

${ }^{5}$ Murch, A.M. and Foster, J.V. "Recent NASA Research on Aerodynamic Modeling of Post-Stall and Spin Dynamics of Large Transport Airplanes", AIAA-2007-463-253, 45 th AIAA Aerospace Sciences Meeting and Exhibit, Reno, NV, January 2007.

${ }^{6}$ Garza, F.R. and Morelli, E.A. "A Collection of Nonlinear Aircraft Simulations in MATLAB," NASA / TM-2003-212145, January 2003.

${ }^{7}$ Engelund, W.C., Holland, S.D., Cockrell, C.E., Jr., Bittner, R.D. “Aerodynamic Database Development for the Hyper-X Airframe Integrated Scramjet Propulsion Experiment," Journal of Spacecraft and Rockets, Vol. 38, No. 6, Nov-Dec 2001, pp. 803-810.

${ }^{8}$ Shah, G.H., Cunningham, K., Foster, J.V., Fremaux, C.M., Stewart, E.C., Wilborn, J.E., Gato, W., Pratt, D.W., "WindTunnel Investigation of Commercial Transport Aircraft Aerodynamics at Extreme Flight Conditions", SAE 2002-01-2912, World Aviation Congress \& Display, Phoenix, AZ, November 2002. 
${ }^{9}$ Stevenson, S.W., Holl, D., and Roman, A. "Parameter Identification of AV-8B Wingborne Aerodynamics for Flight Simulator Model Updates," AIAA-92-4506-CP, AIAA Atmospheric Flight Mechanics Conference, Hilton Head, SC, August 1992.

${ }^{10}$ Jategaonkar, R.V. and Thielecke, F. "Aircraft Parameter Estimation - A Tool for Development of Aerodynamic Databases," Sadhana, Vol. 25, Part 2, April 2000, pp. 119-135.

${ }^{11}$ Morelli, E.A. and Ward, D.G. "Automated Simulation Updates based on Flight Data," AIAA-2007-6714, AIAA Atmospheric Flight Mechanics Conference, Hilton Head, SC, August 2007.

${ }^{12}$ Brian, G. and Morelli, E.A. "Rapid Automated Aircraft Simulation Model Updating From Flight Data," AIAC14, Session 5A, Fourteenth Australian International Aerospace Congress, Melbourne, Australia, March 2011.

${ }^{13}$ Morelli, E.A. "Multiple Input Design for Real-Time Parameter Estimation in the Frequency Domain," Paper REG-360, $13^{\text {th }}$ IFAC Symposium on System Identification, Rotterdam, The Netherlands, August 2003.

${ }^{14}$ Klein, V. and Morelli, E.A., Aircraft System Identification - Theory and Practice, AIAA Education Series, AIAA, Reston, VA, 2006.

${ }^{15}$ Morelli, E.A. "Flight-Test Experiment Design for Characterizing Stability and Control of Hypersonic Vehicles," Journal of Guidance, Control, and Dynamics, Vol. 32, No. 3, May-June 2009, pp. 949-959.

${ }^{16}$ Morelli, E.A. "Flight Test Maneuver Design for Efficient Aerodynamic Modeling," AIAA 2011-6672, AIAA Atmospheric Flight Mechanics Conference, Portland, OR, August 2011.

${ }^{17}$ Morelli, E.A. and Smith, M.S. "Real-Time Dynamic Modeling - Data Information Requirements and Flight Test Results," Journal of Aircraft, Vol. 46, No. 6, November-December 2009, pp. 1894-1905.

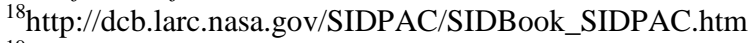

${ }^{19}$ Press, W.H., S.A. Teukolsky, W.T. Vettering, and B.R. Flannery Numerical Recipes in FORTRAN: The Art of Scientific Computing, $2^{\text {nd }}$ Ed., Cambridge University Press, New York, NY, 1992, Chapter 10.

${ }^{20}$ Morelli, E.A. "Global Nonlinear Aerodynamic Modeling using Multivariate Orthogonal Functions," Journal of Aircraft, Vol. 32, No. 2, March-April 1995, pp. 270-77.

${ }^{21}$ Seher-Weiss, S. "Identification of Nonlinear Aerodynamic Derivatives using Classical and Extended Local Model Networks," Aerospace Science and Technology, Vol. 15, 2011, pp. 33-44.

${ }^{22}$ de Visser, C.C., Mulder, J.A., and Chu, Q.P. "Global Aerodynamic Modeling with Multivariate Splines," AIAA-20087500, AIAA Modeling and Simulation Technologies Conference and Exhibit, Honolulu, HI, August 2008.

${ }^{23}$ Barron, A.R., "Predicted Squared Error : A Criterion for Automatic Model Selection," Self-Organizing Methods in Modeling, Farlow, S.J., Ed., Marcel Dekker, Inc., New York, NY, 1984, pp. 87-104.

${ }^{24}$ Jordan, Thomas L., Langford, William M., Hill, Jeffrey S.; "Airborne Subscale Transport Aircraft Research Testbed: Aircraft Model Development", AIAA 2005-6432, AIAA Guidance, Navigation, and Control Conference and Exhibit, San Francisco, CA, August 2005.

${ }^{25}$ Jordan, Thomas L., Foster, John V., Bailey, Roger M., and Belcastro, Christine M., “AirSTAR: A UAV Platform for Flight Dynamics and Control System Testing," AIAA-2006-3307, 25th AIAA Aerodynamic Measurement Technology and Ground Testing Conference, San Francisco, CA, June 2006.

${ }^{26}$ Murch, A. M., "A Flight Control System Architecture for the NASA AirSTAR Flight Test Facility," AIAA 2008-6990, AIAA Guidance, Navigation, and Control Conference and Exhibit, Honolulu, HI, 2008. 
Table 1 Multiple input design for the T-2 subscale jet transport aircraft, level flight, $V_{o}=80 \mathrm{kts}, \alpha_{o}=5 \mathrm{deg}, T=20 \mathrm{sec}$

\begin{tabular}{|c|c|c|c|c|c|}
\hline Input & $A(\operatorname{deg})$ & $A_{k}(\operatorname{deg})$ & $k$ & $\phi_{k}(\mathrm{rad})$ & $R P F$ \\
\hline \multirow{10}{*}{$\delta_{e}$} & \multirow{10}{*}{1.0} & 0.3162 & 5 & -2.2926 & \multirow{10}{*}{1.13} \\
\hline & & 0.3162 & 8 & 0.6842 & \\
\hline & & 0.3162 & 11 & -0.3288 & \\
\hline & & 0.3162 & 14 & 2.1677 & \\
\hline & & 0.3162 & 17 & -2.8795 & \\
\hline & & 0.3162 & 20 & -0.0447 & \\
\hline & & 0.3162 & 23 & -2.8485 & \\
\hline & & 0.3162 & 26 & -2.8634 & \\
\hline & & 0.3162 & 29 & 3.0356 & \\
\hline & & 0.3162 & 32 & 2.7574 & \\
\hline \multirow{10}{*}{$\delta_{r}$} & \multirow{10}{*}{2.0} & 0.6325 & 6 & 0.9222 & \multirow{10}{*}{1.04} \\
\hline & & 0.6325 & 9 & 0.7188 & \\
\hline & & 0.6325 & 12 & 2.8103 & \\
\hline & & 0.6325 & 15 & -0.9035 & \\
\hline & & 0.6325 & 18 & 0.1563 & \\
\hline & & 0.6325 & 21 & -1.8697 & \\
\hline & & 0.6325 & 24 & -0.8279 & \\
\hline & & 0.6325 & 27 & -2.0493 & \\
\hline & & 0.6325 & 30 & 1.1970 & \\
\hline & & 0.6325 & 33 & 0.5219 & \\
\hline \multirow{10}{*}{$\delta_{a}$} & \multirow{10}{*}{1.0} & 0.3162 & 4 & 1.8549 & \multirow{10}{*}{1.17} \\
\hline & & 0.3162 & 7 & -2.6561 & \\
\hline & & 0.3162 & 10 & -2.8832 & \\
\hline & & 0.3162 & 13 & 0.1226 & \\
\hline & & 0.3162 & 16 & 2.5070 & \\
\hline & & 0.3162 & 19 & 2.6150 & \\
\hline & & 0.3162 & 22 & 0.6119 & \\
\hline & & 0.3162 & 25 & -1.9709 & \\
\hline & & 0.3162 & 28 & -1.3854 & \\
\hline & & 0.3162 & 31 & -3.0152 & \\
\hline
\end{tabular}


Table 2. T-2 maneuvers applying multi-axis excitation for slow approach to stall and recovery

\begin{tabular}{|c|c|c|c|}
\hline \hline Configuration & Power Level & Flight & Card \\
\hline Cruise & IDLE & 5 & 10 \\
\hline Cruise & 40 percent & 6 & 11 \\
\hline Cruise & 50 percent & 6 & 12 \\
\hline Cruise & 60 percent & 6 & 13 \\
\hline Takeoff & IDLE & 6 & 15 \\
\hline Cruise & IDLE & 9 & 10 \\
\hline Powered Approach & IDLE & 9 & 17 \\
\hline Powered Approach & IDLE & 11 & 17 \\
\hline Cruise & IDLE & 34 & 17 \\
\hline Cruise & IDLE & 34 & 17 \\
\hline Cruise & IDLE & 36 & 17 \\
\hline Cruise & IDLE & 36 & 17 \\
\hline Cruise & IDLE & 37 & 17 \\
\hline Cruise & IDLE & 37 & 17 \\
\hline Cruise & IDLE & 38 & 17 \\
\hline Cruise & IDLE & 38 & 17 \\
\hline Cruise & IDLE & 38 & 17 \\
\hline Cruise & IDLE & 42 & 17 \\
\hline Cruise & IDLE & 42 & 17 \\
\hline Cruise & IDLE & 42 & 17 \\
\hline
\end{tabular}

Table 3. T-2 aircraft geometry and nominal mass properties

\begin{tabular}{|c|l|}
\hline \hline $\bar{c}, \mathrm{ft}$ & 0.915 \\
\hline$b, \mathrm{ft}$ & 6.849 \\
\hline$S, \mathrm{ft}^{2}$ & 5.902 \\
\hline$x_{o}$, in & 57.30 \\
\hline$y_{o}$, in & 0.000 \\
\hline$z_{o}$, in & 11.28 \\
\hline$x_{c g}$, in & 56.63 \\
\hline$y_{c g}$, in & 0.000 \\
\hline$z_{c g}$, in & 11.43 \\
\hline$m$, slugs & 1.585 \\
\hline$I_{x}$, slugs- $\mathrm{ft}^{2}$ & 1.179 \\
\hline$I_{y}$, slugs- $\mathrm{ft}^{2}$ & 4.520 \\
\hline$I_{z}$, slugs $-\mathrm{ft}^{2}$ & 5.527 \\
\hline$I_{x z}$, slugs- $\mathrm{ft}^{2}$ & 0.211 \\
\hline \hline
\end{tabular}


Table 4. Parameter estimates and uncertainties for $C_{Z}$ global model identified from simulated flight data

\begin{tabular}{|c|c|}
\hline $\begin{array}{c}C_{Z} \text { Model } \\
\text { Parameter }\end{array}$ & $\begin{array}{c}\text { Estimate } \\
\pm \text { Std. Error }\end{array}$ \\
\hline$C_{Z_{o}}$ & $\begin{array}{l}-0.0738 \\
\pm 0.0005\end{array}$ \\
\hline$C_{Z_{\alpha}}$ & -4.4809 \\
& \pm 0.0047 \\
\hline$C_{Z_{q}}$ & -47.241 \\
& \pm 0.2017 \\
\hline$C_{Z_{\delta_{e}}}$ & 0.4500 \\
& \pm 0.0062 \\
\hline$C_{Z_{\alpha_{10}^{1}}}$ & 2.1745 \\
& \pm 0.0124 \\
\hline$C_{Z_{\alpha_{12}^{1}}}$ & 0.8776 \\
\hline
\end{tabular}


Table 5. Parameter estimates and uncertainties for longitudinal global model identified from T-2 flight data

\begin{tabular}{|c|c|c|c|c|c|}
\hline $\begin{array}{l}C_{X} \text { Model } \\
\text { Parameter }\end{array}$ & $\begin{array}{c}\text { Estimate } \\
\pm \text { Std. Error }\end{array}$ & $\begin{array}{l}C_{Z} \text { Model } \\
\text { Parameter }\end{array}$ & $\begin{array}{c}\text { Estimate } \\
\pm \text { Std. Error }\end{array}$ & $\begin{array}{l}C_{m} \text { Model } \\
\text { Parameter }\end{array}$ & $\begin{array}{c}\text { Estimate } \\
\pm \text { Std. Error }\end{array}$ \\
\hline$C_{X_{o}}$ & $\begin{array}{l}-0.1017 \\
\pm 0.0039\end{array}$ & $C_{Z_{o}}$ & $\begin{array}{l}-0.1353 \\
\pm 0.0037\end{array}$ & $C_{m_{\delta_{e}}}$ & $\begin{array}{l}-0.8377 \\
\pm 0.0822\end{array}$ \\
\hline$C_{X_{\alpha}}$ & $\begin{array}{c}1.6921 \\
\pm 0.0754\end{array}$ & $C_{Z_{\alpha}}$ & $\begin{array}{l}-4.0013 \\
\pm 0.0409\end{array}$ & $C_{m_{\alpha^{2}}}$ & $\begin{array}{c}6.5387 \\
\pm 0.6531\end{array}$ \\
\hline$C_{X_{\alpha^{2}}}$ & $\begin{array}{l}-4.4473 \\
\pm 0.3301\end{array}$ & $C_{Z_{\alpha_{6}^{1}}}$ & $\begin{array}{c}1.0897 \\
\pm 0.0488\end{array}$ & $C_{m \alpha \alpha_{8}^{1}}$ & $\begin{array}{l}-1.7790 \\
\pm 0.6789\end{array}$ \\
\hline$C_{X_{q \delta_{e}}}$ & $\begin{array}{l}-306.56 \\
\pm 15.289\end{array}$ & $C_{Z_{q \alpha_{12}^{1}}}$ & $\begin{array}{c}2.0933 \mathrm{e}+03 \\
\pm 134.16\end{array}$ & $C_{m_{q}}$ & $\begin{array}{c}-36.3742 \\
\pm 4.9737\end{array}$ \\
\hline$C_{X}$ & $\begin{array}{l}-806.31 \\
\pm 53.991\end{array}$ & $C_{Z_{q \delta_{e}}}$ & $\begin{array}{c}214.2068 \\
\pm 15.328\end{array}$ & $C_{m_{o}}$ & $\begin{array}{c}0.1592 \\
\pm 0.0059\end{array}$ \\
\hline$C_{X_{\delta_{e}}}$ & $\begin{array}{c}0.3982 \\
\pm 0.0469\end{array}$ & & & $C_{m_{\alpha}}$ & $\begin{array}{l}-2.6947 \\
\pm 0.1243\end{array}$ \\
\hline$C_{X_{\alpha \delta_{e}}}$ & $\begin{array}{l}-1.7624 \\
\pm 0.2961\end{array}$ & & & $C_{m_{q \delta_{e}}}$ & $\begin{array}{c}388.9319 \\
\pm 34.404\end{array}$ \\
\hline$C_{X_{\alpha_{10}^{1}}}$ & $\begin{array}{c}2.2999 \\
\pm 0.1708\end{array}$ & & & $C_{m_{q \alpha_{12}^{1}}}$ & $\begin{array}{c}1.4879 \mathrm{e}+03 \\
\pm 175.32\end{array}$ \\
\hline \multirow[t]{5}{*}{$C_{X_{\alpha \alpha_{6}^{1}}}$} & $\begin{array}{l}-7.9305 \\
\pm 0.6953\end{array}$ & & & $C_{m_{\alpha q}}$ & $\begin{array}{c}237.4375 \\
\pm 50.250\end{array}$ \\
\hline & & & & $C_{m_{\delta_{e} \alpha_{12}^{1}}}$ & $\begin{array}{c}2.7147 \\
\pm 0.6377\end{array}$ \\
\hline & & & & $C_{m}$ & $\begin{array}{l}-198.14 \\
\pm 73.932\end{array}$ \\
\hline & & & & $C_{m_{\alpha \delta_{e}}}$ & $\begin{array}{l}-4.3485 \\
\pm 0.7122\end{array}$ \\
\hline & & & & $C_{m_{\delta_{e} \alpha_{8}^{1}}}$ & $\begin{array}{c}5.5804 \\
\pm 1.1554\end{array}$ \\
\hline
\end{tabular}


Table 6. Parameter estimates and uncertainties for lateral global model identified from T-2 flight data

\begin{tabular}{|c|c|c|c|c|c|}
\hline $\begin{array}{l}C_{Y} \text { Model } \\
\text { Parameter }\end{array}$ & $\begin{array}{c}\text { Estimate } \\
\pm \text { Std. Error }\end{array}$ & $\begin{array}{l}C_{l} \text { Model } \\
\text { Parameter }\end{array}$ & $\begin{array}{c}\text { Estimate } \\
\pm \text { Std. Error }\end{array}$ & $\begin{array}{l}C_{n} \text { Model } \\
\text { Parameter }\end{array}$ & $\begin{array}{l}\text { Estimate } \\
\pm \text { Std. Error }\end{array}$ \\
\hline$C_{Y_{\beta}}$ & $\begin{array}{l}-0.5483 \\
\pm 0.0422\end{array}$ & $C_{l_{\delta_{a}}}$ & $\begin{array}{l}-0.0351 \\
\pm 0.0009\end{array}$ & $C_{n_{\beta}}$ & $\begin{array}{c}0.1673 \\
\pm 0.0104\end{array}$ \\
\hline$C_{Y_{\alpha}}$ & $\begin{array}{c}0.0890 \\
\pm 0.0051\end{array}$ & $C_{l_{r \delta_{a}}}$ & $\begin{array}{l}-0.6816 \\
\pm 0.0690 \\
\end{array}$ & $C_{n_{o}}$ & $\begin{array}{l}-0.0017 \\
\pm 0.0002 \\
\end{array}$ \\
\hline$C_{Y_{\alpha \delta_{r}}}$ & $\begin{array}{c}0.5875 \\
\pm 0.1537\end{array}$ & $C_{l_{p r}}$ & $\begin{array}{l}-2.3733 \\
\pm 0.5028\end{array}$ & $C_{n_{\delta_{r}}}$ & $\begin{array}{l}-0.1705 \\
\pm 0.0020\end{array}$ \\
\hline$C_{Y_{r \delta_{a}}}$ & $\begin{array}{l}-3.7945 \\
\pm 0.5046 \\
\end{array}$ & $C_{l_{r}}$ & $\begin{array}{c}0.2851 \\
\pm 0.0282 \\
\end{array}$ & $C_{n_{\alpha r \delta_{a}}}$ & $\begin{array}{c}5.6599 \\
\pm 1.0124 \\
\end{array}$ \\
\hline$C_{Y_{r}}$ & $\begin{array}{c}2.4864 \\
\pm 0.0528\end{array}$ & $C_{l_{\alpha \alpha_{10}^{1}}}$ & $\begin{array}{c}1.6212 \\
\pm 0.0717\end{array}$ & $C_{n_{r}}$ & $\begin{array}{l}-0.8890 \\
\pm 0.0466\end{array}$ \\
\hline$C_{Y_{p r}}$ & $\begin{array}{c}6.3526 \\
\pm 3.0915\end{array}$ & $C_{l_{\beta}}$ & $\begin{array}{l}-0.1301 \\
\pm 0.0046\end{array}$ & $C_{n_{\alpha p \delta_{a}}}$ & $\begin{array}{l}-1.3790 \\
\pm 0.3426\end{array}$ \\
\hline$C_{Y_{p \delta_{a}}}$ & $\begin{array}{c}1.2747 \\
\pm 0.1657 \\
\end{array}$ & $C_{l_{\alpha}}$ & $\begin{array}{c}5.6202 \mathrm{e}-03 \\
\pm 0.0007\end{array}$ & $C_{n_{\alpha}}$ & $\begin{array}{l}-0.0170 \\
\pm 0.0013\end{array}$ \\
\hline$C_{Y_{\alpha p}}$ & $\begin{array}{c}-10.1120 \\
\pm 0.5171\end{array}$ & $C_{l_{p}}$ & $\begin{array}{l}-0.3172 \\
\pm 0.0059\end{array}$ & $C_{n_{\alpha p}}$ & $\begin{array}{c}2.8815 \\
\pm 0.1457\end{array}$ \\
\hline$C_{Y_{\alpha \delta_{a}}}$ & $\begin{array}{l}-2.3867 \\
\pm 0.0928\end{array}$ & $C_{l_{\delta_{r}}}$ & $\begin{array}{c}0.0392 \\
\pm 0.0011\end{array}$ & $C_{n_{\alpha \delta_{a}}}$ & $\begin{array}{c}0.3648 \\
\pm 0.0221\end{array}$ \\
\hline$C_{Y_{\beta \alpha}}$ & $\begin{array}{l}-4.0360 \\
\pm 0.2620 \\
\end{array}$ & $C_{l_{10}^{1}}$ & $\begin{array}{l}-0.3198 \\
\pm 0.0164 \\
\end{array}$ & $C_{n_{p}}$ & $\begin{array}{l}-0.4471 \\
\pm 0.0275 \\
\end{array}$ \\
\hline$C_{Y_{\delta_{r}}}$ & $\begin{array}{c}0.2479 \\
\pm 0.0256\end{array}$ & $C_{r \alpha_{10}^{1}}$ & $\begin{array}{c}3.2163 \\
\pm 0.5092\end{array}$ & $C_{n_{\delta_{a}}}$ & $\begin{array}{l}-0.0485 \\
\pm 0.0031\end{array}$ \\
\hline$C_{Y_{\beta \delta_{a}}}$ & $\begin{array}{c}2.2167 \\
\pm 0.1807\end{array}$ & $C_{l_{\beta \alpha}}$ & $\begin{array}{l}-0.2710 \\
\pm 0.0293\end{array}$ & $C_{n_{\beta \alpha}}$ & $\begin{array}{c}0.3415 \\
\pm 0.0665\end{array}$ \\
\hline$C_{Y_{\delta_{a}}}$ & $\begin{array}{c}0.2640 \\
\pm 0.0145 \\
\end{array}$ & $C_{l p \alpha_{10}^{1}}$ & $\begin{array}{c}2.0470 \\
\pm 0.1348 \\
\end{array}$ & $C_{n_{\beta \alpha \delta_{a}}}$ & $\begin{array}{l}-3.1717 \\
\pm 0.4148 \\
\end{array}$ \\
\hline$C_{Y_{p}}$ & $\begin{array}{c}1.2558 \\
\pm 0.1045\end{array}$ & $C_{p \alpha_{14}^{1}}$ & $\begin{array}{l}-8.4945 \\
\pm 0.7066\end{array}$ & $C_{n_{\alpha r}}$ & $\begin{array}{c}1.9498 \\
\pm 0.3031\end{array}$ \\
\hline$C_{Y_{o}}$ & $\begin{array}{c}5.3615 \mathrm{e}-03 \\
\pm 0.0007\end{array}$ & $C_{l_{p \delta_{a}}}$ & $\begin{array}{c}0.1441 \\
\pm 0.0176 \\
\end{array}$ & $C_{n_{p \delta_{r}}}$ & $\begin{array}{c}1.0237 \\
\pm 0.1960 \\
\end{array}$ \\
\hline \multirow[t]{3}{*}{$C_{Y_{p \delta_{r}}}$} & $\begin{array}{l}-4.1527 \\
\pm 0.7488 \\
\end{array}$ & $C_{l_{o}}$ & $\begin{array}{c}8.7560 \mathrm{e}-04 \\
\pm 0.0001 \\
\end{array}$ & & \\
\hline & & $C_{l_{\alpha r}}$ & $\begin{array}{c}1.0919 \\
\pm 0.2052 \\
\end{array}$ & & \\
\hline & & $C_{l_{r \delta_{r}}}$ & $\begin{array}{c}1.3430 \\
\pm 0.2878 \\
\end{array}$ & & \\
\hline
\end{tabular}

